\title{
WEIGHTED HOMOGENEOUS SURFACE SINGULARITIES HOMEOMORPHIC TO BRIESKORN COMPLETE INTERSECTIONS
}

\author{
TOMOHIRO OKUMA
}

\begin{abstract}
For a given topological type of a normal surface singularity, there are various types of complex structures which realize it. We are interested in the following problem: Find the maximum of the geometric genus and a condition for that the maximal ideal cycle coincides with the fundamental cycle on the minimal good resolution. In this paper, we study weighted homogeneous surface singularities homeomorphic to Brieskorn complete intersection singularities from the perspective of the problem.
\end{abstract}

\section{INTRODUCTION}

The topological type of a normal surface singularity is determined by its resolution graph ([20]). For a given resolution graph of a normal surface singularity, there are various types of complex structures which realize it. We are interested in finding the upper (resp. lower) bound of basic invariants (e.g., the geometric genus), and in understanding the complex structures which attain their maximum (resp. minimum).

Let $(V, o)$ be a normal complex surface singularity with minimal good resolution $X \rightarrow V$ and let $\Gamma$ be the resolution graph of $(V, o)$. As noticed above, the topological invariants of $(V, o)$ are precisely the invariants of $\Gamma$. In this paper, we consider the geometric genus $p_{g}(V, o)=\operatorname{dim} H^{1}\left(\mathcal{O}_{X}\right)$ and the maximal ideal cycle $M_{X}$ on $X$. In general, these invariants cannot be determined by $\Gamma$ and it is difficult to compute them. By the definition (Definition 2.1), the fundamental cycle $Z_{X}$ on $X$ is determined by $\Gamma$ and the inequality $M_{X} \geq Z_{X}$ holds. The fundamental problem we wish to explore is the following.

Problem 1.1. Let $p_{g}(\Gamma)$ denote the maximum of the geometric genus over the normal surface singularities with resolution graph $\Gamma$.

(1) Find the value $p_{g}(\Gamma)$ and conditions for $M_{X}=Z_{X}$.

(2) Describe the properties and invariants of a singularity $(V, o)$ with $p_{g}(V, o)=$ $p_{g}(\Gamma)$ or $M_{X}=Z_{X}$.

It is known that in a complex analytic family of the resolution space $X$ preserving $\Gamma$ (cf. [10]), the dimension of the cohomology of the structure sheaf is upper semicontinuous. So, we expect the singularities $(V, o)$ with $p_{g}(V, o)=p_{g}(\Gamma)$ may have some kind of nice structure.

2010 Mathematics Subject Classification. Primary 32S25; Secondary 14J17, 32S05, 14 B05.

Key words and phrases. Surface singularities, weighted homogeneous singularities, Brieskorn complete intersections, geometric genus, maximal ideal cycles.

This work was partially supported by JSPS KAKENHI Grant Number 17K05216. 
The equality $M_{X}=Z_{X}$ holds for rational singularities ([2]), minimally elliptic singularities ([9]), and hypersurfaces $z^{n}=f(x, y)$ with certain conditions ([5], [31]). We have an explicit condition for the equality $M_{X}=Z_{X}$ for Brieskorn complete intersection singularities ([8], [12]); the result is generalized to Kummer coverings over weighted homogeneous normal surface singularities in [29]. The upper bound of $p_{g}$ has been also studied by several authors (e.g., [34], [27], [28], [15], [19]); the "rational trees" $\Gamma$ whose $p_{g}(\Gamma)$ can be obtained from $\Gamma$ are listed in $[18,1.7]$. In Example 2.9 of the present paper, we shall introduce the weighted homogeneous singularities of hyperelliptic type for which $p_{g}(\Gamma)$ is easily computed. Since $p_{g}(V, o)=\operatorname{dim} H^{0}\left(\mathcal{O}_{X}\right) / H^{0}\left(\mathcal{O}_{X}\left(-Z_{K_{X}}\right)\right)$ for numerically Gorenstein singularity, where $Z_{K_{X}}$ is the canonical cycle (Definition 2.1), it might be natural to expect that there is a correlation between the properties $p_{g}(V, o)=p_{g}(\Gamma)$ and $M_{X}=Z_{X}$. In fact, when $(V, o)$ is a numerically Gorenstein elliptic singularity (this is characterized by $\Gamma)$, we have that $p_{g}(V, o)=p_{g}(\Gamma)$ if and only if $(V, o)$ is a Gorenstein singularity with $M_{X}=Z_{X}\left([21,5.10]\right.$, [34], [14]); in this case, $p_{g}(\Gamma)$ coincides with the length of the elliptic sequence. However, in [18], we found an example such that the equality $p_{g}=p_{g}(\Gamma)$ is realized by both a Gorenstein singularity with $M_{X}>Z_{X}$ and a non-Gorenstein singularity with $M_{X}=Z_{X}$. In Section 4, we give an example which shows that the condition $M_{X}=Z_{X}$ cannot control $p_{g}$.

In this paper, we study normal surface singularities homeomorphic to Brieskorn complete intersection singularities from the perspective of our problem above. First suppose that $V$ is a complete intersection given as follows:

$$
V=\left\{\left(x_{i}\right) \in \mathbb{C}^{m} \mid q_{i 1} x_{1}^{a_{1}}+\cdots+q_{i m} x_{m}^{a_{m}}=0, \quad i=3, \ldots, m\right\} \quad\left(q_{i j} \in \mathbb{C}\right) .
$$

The resolution graph of the singularity $(V, o)$ is determined by the integers $a_{1}, \ldots, a_{m}$ (Theorem 3.2). We denote it by $\Gamma\left(a_{1}, \ldots, a_{m}\right)$. Using the Pinkham-Demazure divisor $D$ on the central curve $E_{0}$ of the exceptional set $E \subset X$, the homogeneous coordinate ring $R$ of $V$ is represented as $R=\bigoplus_{k \geq 0} H^{0}\left(\mathcal{O}_{E_{0}}\left(D_{k}\right)\right) T^{k}$ (see Section 2.2). We study arithmetic properties of the numerical invariants arising from the topological type in terms of the divisors $D_{k}$ on $E_{0}$. For this purpose, we employ the monomial cycles (cf. [22]) to connect the numerical information of the divisors $D_{k}$ and the complex analytic functions on $X$; note that monomial cycles play an important role in the study of invariants of splice quotients ([22], [16]). For example, we show that $H^{0}\left(\mathcal{O}_{E_{0}}\left(D_{k}\right)\right) \neq 0$ if and only if $\operatorname{deg} D_{k}$ is a member of a certain semigroup, and that $D_{k} \sim D_{k^{\prime}}$ if and only if $\operatorname{deg} D_{k}=\operatorname{deg} D_{k^{\prime}}$ (see Proposition 3.8, Theorem 3.10). Applying these results, we obtain the following (see Theorem 3.9, Theorem 3.12).

Theorem 1.2. If $(V, o)$ is a Brieskorn complete intersection such that the central curve $E_{0}$ is rational or elliptic curve, then $p_{g}(V, o)=p_{g}(\Gamma)$ and $M_{X}=Z_{X}$.

Even if the singularity is not a Brieskorn complete intersection, we can apply a part of the argument on the divisors $D_{k}$ and prove the following (Theorem 3.16).

Theorem 1.3. There exists a weighted homogeneous singularity with resolution graph $\Gamma\left(a_{1}, \ldots, a_{m}\right)$ such that the maximal ideal cycle coincides with the fundamental cycle on the minimal good resolution.

We shall describe the property of the Pinkham-Demazure divisor corresponding to the singularity in Theorem 1.3. 
If the central curve $E_{0}$ has genus $g \geq 2$, we cannot expect a result similar to Theorem 1.2. In fact, there may be various types of complex structures even when $g=2$. To show this, in Section 4 , we fix a resolution graph $\Gamma=\Gamma(2,3,3,4)$, which is the simplest one in a sense, and investigate the singularities having this graph. Any Brieskorn complete intersection singularity with this graph satisfies neither $p_{g}(V, o)=p_{g}(\Gamma)$ nor $M_{X}=Z_{X}$. Assume that $(V, o)$ is a weighted homogeneous surface singularity with resolution graph $\Gamma$. We prove that $(V, o)$ satisfies $p_{g}(V, o)=$ $p_{g}(\Gamma)$ if and only if it is hyperelliptic type, and show that such a singularity is a complete intersection, which is a double cover of a rational double point of type $A_{1}$. For the geometric genus, the multiplicity, and the embedding dimension of these singularities, see Table 1, where the rightmost column indicates the subsections which include the details.

\begin{tabular}{ccccl}
\hline \hline type & $p_{g}$ & mult & embdim & Section \\
\hline Brieskorn CI & 8 & 6 & 4 & Section 4.1 \\
\hline maximal $p_{g}$ & 10 & 4 & 4 & Section 4.2 \\
\hline \hline
\end{tabular}

TABLE 1. Special types

Next, in Section 4.3, we give a complete classification of the weighted homogeneous normal surface singularities $(V, o)$ with resolution graph $\Gamma=\Gamma(2,3,3,4)$ such that $M_{X}=Z_{X}$. We can see the fundamental invariants of those singularities in Table 2. For each class, we prove the existence of the singularities by showing the explicit description of the Pinkham-Demazure divisor (cf. Section 4.3).

\begin{tabular}{ccccl}
\hline \hline$p_{g}$ & mult & embdim & ring & Proposition \\
\hline 8 & 3 & 4 & non Gorenstein & 4.13 \\
8 & 4 & 4 & non Gorenstein & $4.16(1)$ \\
7 & 4 & 5 & non Gorenstein & $4.16(2)$ \\
8 & 5 & 5 & Gorenstein & $4.18(1)$ \\
7 & 5 & 5 & non Gorenstein & $4.18(2)$ \\
6 & 6 & 7 & non Gorenstein & 4.21 \\
\hline \hline
\end{tabular}

TABLE 2. Singularities with $M_{X}=Z_{X}$

Note that for any two singularities in Table 2, they have the same thick-thin decomposition if and only if they have the same multiplicity; see [4] and the proof of Proposition 4.10 (2).

This paper is organized as follows. In Section 2, we review basic facts on weighted homogeneous surface singularities and introduce the singularity of hyperelliptic type. In Section 3, first we summarize the results on Brieskorn complete intersection surface singularities, and prove Theorem 1.2 and Theorem 1.3. In Section 4, we study weighted homogeneous singularities with resolution graph $\Gamma=\Gamma(2,3,3,4)$ such that $p_{g}=p_{g}(\Gamma)$ and those with $M_{X}=Z_{X}$. 
Acknowledgement. The author would like to thank the referee for reading the paper carefully and providing several thoughtful comments which helped improving the paper, especially, Lemma 4.3 and Proposition 4.9.

\section{Preliminaries}

Let $(V, o)$ be a normal complex surface singularity, namely, the germ of a normal complex surface $V$ at $o \in V$. We denote by $\mathfrak{m}$ the maximal ideal of the local ring $\mathcal{O}_{V, o}$. Let $\pi: X \rightarrow V$ denote the minimal good resolution of the singularity $(V, o)$ with exceptional set $E=\pi^{-1}(p)$, and let $\left\{E_{i}\right\}_{i \in \mathcal{I}}$ denote the set of irreducible components of $E$. We denote by $\Gamma$ the resolution graph of $(V, o)$, namely, the weighted dual graph of $E$. A divisor on $X$ supported in $E$ is called a cycle. We denote the group of cycles by $\mathbb{Z} E$. An element of $\mathbb{Q} E:=\mathbb{Z} E \otimes \mathbb{Q}$ is called a $\mathbb{Q}$-cycle. Since the intersection matrix $\left(E_{i} E_{j}\right)$ is negative definite, for every $j \in \mathcal{I}$ there exists an effective $\mathbb{Q}$-cycle $E_{j}^{*}$ such that $E_{j}^{*} E_{i}=-\delta_{j i}$, where $\delta_{j i}$ denotes the Kronecker delta. Let $\mathbb{Z} E^{*} \subset \mathbb{Q} E$ denote the subgroup generated by $\left\{E_{i}^{*}\right\}_{i \in I}$.

For any $\mathbb{Q}$-divisor $F=\sum c_{i} F_{i}$ with distinct irreducible components $F_{i}$, we denote by $\operatorname{cff}_{F_{i}}(F)$ the coefficient of $F_{i}$ in $F$, i.e., $\operatorname{cff}_{F_{i}}(F)=c_{i}$. For a function $h \in$ $H^{0}\left(\mathcal{O}_{X}\right) \backslash\{0\}$, we denote by $(h)_{E} \in \mathbb{Z} E$ the exceptional part of the $\operatorname{divisor}_{\operatorname{div}_{X}}(h)$; this means that $\operatorname{div}_{X}(h)-(h)_{E}$ is an effective divisor containing no components of $E$. We call $\operatorname{div}_{X}(h)-(h)_{E}$ the non-exceptional part of $\operatorname{div}_{X}(h)$. We simply write $(h)_{E}$ instead of $(h \circ \pi)_{E}$ for $h \in \mathfrak{m} \backslash\{0\}$.

A $\mathbb{Q}$-cycle $D$ is said to be $n e f$ (resp. anti-nef) if $D E_{i} \geq 0$ (resp. $D E_{i} \leq 0$ ) for all $i \in \mathcal{I}$. Note that if a cycle $D \neq 0$ is anti-nef, then $D \geq E$.

Definition 2.1. The fundamental cycle is by definition the smallest non-zero antinef cycle and denoted by $Z_{X}$. The maximal ideal cycle on $X$ is the minimum of $\left\{(h)_{E} \mid h \in \mathfrak{m} \backslash\{0\}\right\}$ and denoted by $M_{X}$. Clearly, $Z_{X} \leq M_{X}$. There exists a $\mathbb{Q}$ cycle $Z_{K_{X}}$ such that $\left(K_{X}+Z_{K_{X}}\right) E_{i}=0$ for every $i \in \mathcal{I}$, where $K_{X}$ is a canonical divisor on $X$. We call $Z_{K_{X}}$ the canonical cycle on $X$.

2.1. Cyclic quotient singularities. Let $n$ and $\mu$ be positive integers with $\mu<n$ and $\operatorname{gcd}(n, \mu)=1$. Let $\epsilon_{n} \in \mathbb{C}$ denote the primitive $n$-th root of unity and let $G$ denote the cyclic group $\left\langle\left(\begin{array}{cc}\epsilon_{n} & 0 \\ 0 & \epsilon_{n}^{\mu}\end{array}\right)\right\rangle \subset G L(2, \mathbb{C})$. Suppose that $V=\mathbb{C}^{2} / G$. Then $(V, o)$ is called the cyclic quotient singularity of type $C_{n, \mu}$. For integers $c_{i} \geq 2$, $i=1, \ldots, r$, we put

$$
\left[\left[c_{1}, \ldots, c_{r}\right]\right]:=c_{1}-\frac{1}{c_{2}-\frac{1}{\ddots \cdot-\frac{1}{c_{r}}}}
$$

If $n / \mu=\left[\left[c_{1}, \ldots, c_{r}\right]\right]$, the resolution graph $\Gamma$ is a chain as in Figure 1, where all components $E_{i}$ are rational.

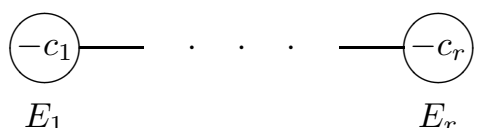

FIGURE 1. The resolution graph of $C_{n, \mu}$ 
It is known that the local class group $\operatorname{Cl}(V, o)$ is isomorphic to the finite abelian group

$$
\mathbb{Z} E^{*} / \mathbb{Z} E=\left\langle\left[E_{1}^{*}\right]\right\rangle=\left\langle\left[E_{r}^{*}\right]\right\rangle
$$

of order $n$, where $\left[E_{i}^{*}\right]=E_{i}^{*}+\mathbb{Z} E$ (cf. [13, II (a)], [3, III. 5]).

Suppose that $E_{0}$ is a prime divisor on $X$ such that $E_{0} E_{i}=\delta_{1 i}$ for $1 \leq i \leq r$; so $E_{0}+E_{1}+\cdots+E_{r}$ looks like a chain of curves. For any positive integer $m_{0}$, let

$$
\mathcal{L}\left(m_{0}\right)=\left\{m_{0} E_{0}+\sum_{i=1}^{r} m_{i} E_{i} \mid m_{1}, \ldots, m_{r} \in \mathbb{Z}_{>0}\right\} .
$$

Then we define a set $\mathcal{D}\left(m_{0}\right)$ as follows:

$$
\mathcal{D}\left(m_{0}\right):=\left\{D \in \mathcal{L}\left(m_{0}\right) \mid D E_{i} \leq 0, i=1, \ldots, r\right\} .
$$

It is easy to see that $\mathcal{D}\left(m_{0}\right)$ is not empty and has a unique smallest element.

Let $\lceil x\rceil$ denote the ceiling of a real number $x$.

Lemma 2.2. Let $D \in \mathcal{D}\left(m_{0}\right)$. We have the following:

(1) There exists an effective cycle $F$ such that $(D+F) E_{i}=0$ for $1 \leq i<r$ and $\operatorname{Supp}(F) \subset \bigcup_{i>1} E_{i}$.

(2) If $D E_{i}=0$ for $1 \leq i<r$ and $D E_{r} \geq-1$, then $D$ is the smallest element of $\mathcal{D}\left(m_{0}\right)$.

(3) Assume that $D, D^{\prime} \in \mathcal{D}\left(m_{0}\right)$ and $D E_{i}=D^{\prime} E_{i}$ for $1 \leq i<r$. If $D>D^{\prime}$, then $\operatorname{cff}_{E_{1}}(D)>\operatorname{cff}_{E_{1}}\left(D^{\prime}\right)$.

(4) Assume that $D$ and $D^{\prime}$ are the smallest elements of $\mathcal{D}\left(m_{0}\right)$ and $\mathcal{D}\left(m_{0}^{\prime}\right)$, respectively, and that $D^{\prime} E_{i}=0$ for $1 \leq i \leq r$. Then $D+D^{\prime}$ is the smallest element of $\mathcal{D}\left(m_{0}+m_{0}^{\prime}\right)$.

Proof. We write as $D=\sum_{i=0}^{r} m_{i} E_{i}$ and $D^{\prime}=\sum_{i=0}^{r} m_{i}^{\prime} E_{i}$.

(1) For any $1 \leq k<r$, there exists a cycle $F^{\prime}$ supported on $E_{k+1}+\cdots+E_{r}$ such that

$$
\operatorname{cff}_{E_{k+1}}\left(F^{\prime}\right)=1, \quad F^{\prime} E_{k+1}=\cdots=F^{\prime} E_{r-1}=0, \quad F^{\prime} E_{r}<0
$$

(cf. [3, III.5]). If $a:=D E_{k}<0$, then $D+a F^{\prime} \in \mathcal{D}\left(m_{0}\right)$ and $\left(D+a F^{\prime}\right) E_{k}=0$. By repeating this process, we obtain the assertion.

(2) It follows from [11, Lemma 2.2] (cf. [12, 2.1]).

(3) If $m_{1}=m_{1}^{\prime}$, we can take $1 \leq k<r$ so that $m_{i}=m_{i}^{\prime}$ for $i \leq k$ and $m_{k+1}>$ $m_{k+1}^{\prime}$. Then $\left(D-D^{\prime}\right) E_{k}=m_{k+1}-m_{k+1}^{\prime}>0$; it contradicts that $D E_{k}=D^{\prime} E_{k}$.

(4) Let $d_{i}=\left[\left[c_{i}, \ldots, c_{r}\right]\right]$. By $[8$, Lemma 1.1], the minimality of $D$ is characterized by the condition that $m_{i}=\left\lceil m_{i-1} / d_{i}\right\rceil$ for $1 \leq i \leq r$. By the assumption, it follows from Lemma $1.2(1)$ and (2) of [8] that $m_{i}^{\prime}=m_{i-1}^{\prime} / d_{i}$. Hence we have $m_{i}+m_{i}^{\prime}=\left\lceil m_{i-1} / d_{i}\right\rceil+m_{i-1}^{\prime} / d_{i}=\left\lceil\left(m_{i-1}+m_{i-1}^{\prime}\right) / d_{i}\right\rceil$.

2.2. Weighted homogeneous surface singularities. Let us recall some fundamental facts on weighted homogeneous surface singularities (cf. [23]).

Assume that $(V, o)$ is a weighted homogeneous singularity. Then the resolution graph $\Gamma$ of $(V, o)$ is a star-shaped graph as in Figure 2, where $E_{i, j}$ are rational curves, $g$ is the genus of the curve $E_{0}, c_{i, j}$ and $c_{0}$ are the self-intersection numbers. The component $E_{0}$ is called the central curve.

For $1 \leq i \leq m$, we define positive integers $\alpha_{i}$ and $\beta_{i}$ with $\operatorname{gcd}\left(\alpha_{i}, \beta_{i}\right)=1$ by $\alpha_{i} / \beta_{i}=\left[\left[c_{i, 1}, \ldots, c_{i, s_{i}}\right]\right]$. The data

$$
\left(g, c_{0},\left(\alpha_{1}, \beta_{1}\right), \ldots,\left(\alpha_{m}, \beta_{m}\right)\right)
$$




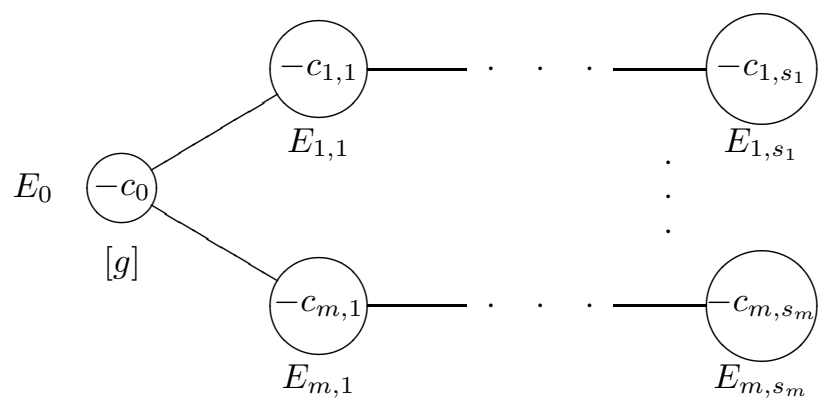

FiguRE 2. A star-shaped resolution graph

is called the Seifert invariant. Note that the graph $\Gamma$ can be recovered from the Seifert invariant.

Let $P_{i} \in E_{0}$ denote the point $E_{0} \cap E_{i, 1}$ and $Q$ a divisor on $E_{0}$ such that $\mathcal{O}_{E_{0}}\left(-E_{0}\right) \cong \mathcal{O}_{E_{0}}(Q)$. We define a $\mathbb{Q}$-divisor $D$ and divisors $D_{k}\left(k \in \mathbb{Z}_{\geq 0}\right)$ on $E_{0}$ as follows:

$$
D:=Q-\sum_{i=1}^{m} \frac{\beta_{i}}{\alpha_{i}} P_{i}, \quad D_{k}:=k Q-\sum_{i=1}^{m}\left\lceil\frac{k \beta_{i}}{\alpha_{i}}\right\rceil P_{i} .
$$

We call $D$ the Pinkham-Demazure divisor. It is known that $\operatorname{deg} D>0$. For any divisor $F$ on $E_{0}$, we write as

$$
H^{i}(F)=H^{i}\left(\mathcal{O}_{E_{0}}(F)\right), \quad h^{i}(F)=\operatorname{dim}_{\mathbb{C}} H^{i}(F) .
$$

Let $R:=R(V, o)$ denote the homogeneous coordinate ring of the singularity $(V, o)$. Then we have the expression $R=\bigoplus_{k \geq 0} H^{0}\left(D_{k}\right) T^{k} \subset \mathbb{C}\left(E_{0}\right)[T]$, where $\mathbb{C}\left(E_{0}\right)$ is the field of rational functions on $E_{0}$ and $T$ an indeterminate (cf. [23], $[30])$. We have the following.

Theorem 2.3 (Pinkham [23]). $p_{g}(V, o)=\sum_{k \geq 0} h^{1}\left(D_{k}\right)$.

Let $H(V, t)$ denote the Hilbert series of the graded ring $R$, i.e., $H(V, t)=$ $\sum_{k \geq 0} h^{0}\left(D_{k}\right) t^{k}$

Proposition 2.4. We have the following.

(1) If we write as $H(V, t)=p(t) / q(t)+r(t)$, where $p, q, r \in \mathbb{C}[t]$ and $\operatorname{deg} p<$ $\operatorname{deg} q$, then $p_{g}(V, o)=r(1)$.

(2) Let $\left(V_{1}, o_{1}\right)$ and $\left(V_{2}, o_{2}\right)$ be weighted homogeneous singularities with the same resolution graph. Then $p_{g}\left(V_{1}, o_{1}\right)-p_{g}\left(V_{2}, o_{2}\right)=\left.\left(H\left(V_{1}, t\right)-H\left(V_{2}, t\right)\right)\right|_{t=1}$.

Proof. (1) follows from [17, 3.1.3].

(2) It follows from Theorem 2.3 and the Riemann-Roch theorem $h^{0}\left(D_{n}\right)-$ $h^{1}\left(D_{n}\right)=\operatorname{deg} D_{n}+1-g$ (the right-hand side is determined by $\Gamma$ ).

The next theorem follows from [33, 2.9].

Theorem 2.5. Let $D^{\prime}=\sum\left(\left(\alpha_{i}-1\right) / \alpha_{i}\right) P_{i}$. Then $R$ is Gorenstein if and only if there exists an integer a such that $K_{C} \sim a D-D^{\prime}$; the integer a coincides with the a-invariant a $(R)$ of Goto-Watanabe ([6]). 
2.3. Surface singularities with star-shaped graph. First, we briefly review some important facts in $[30, \S 6]$. Assume that $(V, o)$ is a normal surface singularity with star-shaped resolution graph $\Gamma$ as Figure 2. Even if $(V, o)$ is not weighted homogeneous, in the same manner as in Section 2.2, we obtain the Pinkham-Demazure divisor

$$
D=Q-\sum_{i=1}^{m} \frac{\beta_{i}}{\alpha_{i}} P_{i}
$$

on the central curve $E_{0} \subset E$ on the minimal good resolution $X$. We define the graded ring $R$ by

$$
R=R\left(E_{0}, D\right):=\bigoplus_{k \geq 0} H^{0}\left(D_{k}\right) T^{k} \subset \mathbb{C}\left(E_{0}\right)[T] .
$$

Let $\bar{V}=\operatorname{Spec} R$ and $o \in \bar{V}$ the point defined by the maximal ideal $\bigoplus_{k \geq 1} H^{0}\left(D_{k}\right) T^{k}$. Then $(\bar{V}, o)$ is a weighted homogeneous normal surface singularity with resolution graph $\Gamma$.

Theorem 2.6 (Tomari-Watanabe $[30, \S 6])$. For every $n \in \mathbb{Z}_{\geq 0}$, there exists the minimal cycle $L_{n} \in \mathbb{Z} E$ such that $L_{n}$ is anti-nef on $E-E_{0}$ and $\operatorname{cff}_{E_{0}}\left(L_{n}\right)=n .{ }^{1}$ Then we have a natural isomorphism $\mathcal{O}_{E_{0}}\left(-L_{n}\right) \cong \mathcal{O}_{E_{0}}\left(D_{n}\right)$ for $n \in \mathbb{Z}_{\geq 0}$; in fact,

$$
\sum_{i=1}^{m}\left\lceil\frac{k \beta_{i}}{\alpha_{i}}\right\rceil P_{i}=\left.\left(L_{n}-n E_{0}\right)\right|_{E_{0}} .
$$

In general, we have $p_{g}(V, o) \leq p_{g}(\bar{V}, o)$. If the equality $p_{g}(V, o)=p_{g}(\bar{V}, o)$ holds, the following sequence is exact for $n \geq 0$ :

$$
0 \rightarrow H^{0}\left(\mathcal{O}_{X}\left(-L_{n}-E_{0}\right)\right) \rightarrow H^{0}\left(\mathcal{O}_{X}\left(-L_{n}\right)\right) \rightarrow H^{0}\left(\mathcal{O}_{E_{0}}\left(D_{n}\right)\right) \rightarrow 0 .
$$

Remark 2.7. From the definitions of $Z_{X}$ and $M_{X}$, we have the following:

$$
\begin{aligned}
\operatorname{cff}_{E_{0}}\left(Z_{X}\right) & =\min \left\{m \in \mathbb{Z}_{>0} \mid \operatorname{deg} D_{m} \geq 0\right\}, \\
\operatorname{cff}_{E_{0}}\left(M_{X}\right) & =\min \left\{m \in \mathbb{Z}_{>0} \mid H^{0}\left(D_{m}\right) \neq 0\right\} .
\end{aligned}
$$

Clearly, $z_{0}:=\operatorname{cff}_{E_{0}}\left(Z_{X}\right) \leq m_{0}:=\operatorname{cff}_{E_{0}}\left(M_{X}\right)$. One of fundamental problems is to find a characterization for the equality $z_{0}=m_{0}$. We have $Z_{X}=L_{z_{0}}$ by the definition of the cycles $L_{n}$. It might be natural to ask whether the condition $m_{0}=z_{0}$ implies the equality $M_{X}=Z_{X}$. For Brieskorn complete intersection singularities, we have a criterion for $z_{0}=m_{0}$ and we always have $M_{X}=L_{m_{0}}$ (see [8], [12]). However, in general, this is not true even for weighted homogeneous singularities (see [29]). We will see later (Proposition 4.9) an example of a weighted homogeneous singularity homeomorphic to a Brieskorn complete intersection singularity which does not satisfy $M_{X}=L_{m_{0}}$ though $z_{0}=m_{0}$ and has the "maximal geometric genus" in the following sense.

Definition 2.8. Let $\mathcal{X}(\Gamma)$ denote the set of normal surface singularities with resolution graph $\Gamma$ and let

$$
p_{g}(\Gamma):=\max \left\{p_{g}(W, o) \mid(W, o) \in \mathcal{X}(\Gamma)\right\} .
$$

\footnotetext{
${ }^{1}$ Our symbol $L_{n}$ is equal to $-L_{-n}$ in $[30, \S 6]$.
} 
Obviously, $p_{g}(\Gamma)$ is an invariant of $\Gamma$. From Theorem 2.6, $p_{g}(\Gamma)$ is attained by a weighted homogeneous singularity. However, the inequality $p_{g}(\bar{V}, o)<p_{g}(\Gamma)$ may happen in general, namely, $p_{g}(\bar{V}, o)$ is not topological, even if $\Gamma$ is a resolution graph of a Brieskorn complete intersection singularity (see Section 4).

Let $\lfloor x\rfloor$ denote the floor (or, integer part) of a real number $x$.

Example 2.9. Assume that a resolution graph $\Gamma$ has the Seifert invariant

$$
\left(g, c_{0}, k_{1}\left(\alpha_{1}, \beta_{1}\right), \ldots, k_{m}\left(\alpha_{m}, \beta_{m}\right)\right) \text {, }
$$

where $k_{i}\left(\alpha_{i}, \beta_{i}\right)$ means that $\left(\alpha_{i}, \beta_{i}\right)$ is repeated $k_{i}$ times, and $\left(\alpha_{i}, \beta_{i}\right) \neq\left(\alpha_{j}, \beta_{j}\right)$ for $i \neq j$. Moreover, assume that $k_{2}, \ldots, k_{m} \in 2 \mathbb{Z}$; in this case, we call $\Gamma$ a hyperelliptic type.

Let $C$ be a hyperelliptic or elliptic curve of genus $g$ and let $\mathcal{R}(C)$ be the set of ramification points of the double cover $C \rightarrow \mathbb{P}^{1}$ with involution $\sigma: C \rightarrow C$. Let $P \in \mathcal{R}(C)$ and $Q=c_{0} P$. Take $P_{i, j} \in C \backslash \mathcal{R}(C)\left(1 \leq i \leq m, 1 \leq j \leq\left\lfloor k_{i} / 2\right\rfloor\right)$ so that $P_{1,1}, \sigma\left(P_{1,1}\right), \ldots, P_{m,\left\lfloor k_{m} / 2\right\rfloor}, \sigma\left(P_{m,\left\lfloor k_{m} / 2\right\rfloor}\right)$ are different from each other. Let $Q_{i, j}=P_{i, j}+\sigma\left(P_{i, j}\right)$. Then we define the Pinkham-Demazure divisor $D$ on $C$ by

$$
D= \begin{cases}Q-\sum_{i=1}^{m} \frac{\beta_{i}}{\alpha_{i}} \sum_{j=1}^{k_{i} / 2} Q_{i, j} & \text { if } k_{1} \in 2 \mathbb{Z}, \\ Q-\frac{\beta_{1}}{\alpha_{1}} P-\frac{\beta_{1}}{\alpha_{1}} \sum_{j=1}^{\left(k_{1}-1\right) / 2} Q_{1, j}-\sum_{i=2}^{m} \frac{\beta_{i}}{\alpha_{i}} \sum_{j=1}^{k_{i} / 2} Q_{i, j} & \text { if } k_{1} \notin 2 \mathbb{Z} .\end{cases}
$$

Since $Q_{i, j} \sim 2 P$, we have $D_{n} \sim\left(\operatorname{deg} D_{n}\right) P$. Let $R=\bigoplus_{k \geq 0} H^{0}\left(D_{k}\right) T^{k}$ and $\bar{V}=$ Spec $R$. We say that the weighted homogeneous normal surface singularity $(\bar{V}, o)$ is a hyperelliptic type, too. Then the singularity $(\bar{V}, o)$ has the resolution graph $\Gamma$ and $p_{g}(\bar{V}, o)=p_{g}(\Gamma)$, because it follows from Clifford's theorem that $h^{1}\left(D_{n}\right)$ is the maximum of $h^{1}\left(D_{n}^{\prime}\right)$, where $C^{\prime}$ is any nonsingular curve of genus $g$ and $D^{\prime}$ is any Pinkham-Demazure divisor on $C^{\prime}$ which corresponding to the resolution graph $\Gamma$.

The following problems are open even for Brieskorn complete intersections.

Problem 2.10. Give an explicit way to compute $p_{g}(\Gamma)$ from $\Gamma$.

Problem 2.11. Classify complex structures which attain $p_{g}(\Gamma)$. Is $E_{0}$ always hyperelliptic if $p_{g}(V, o)=p_{g}(\Gamma)$ ?

Problem 2.12. How can we generalize the notion of "hyperelliptic type" to nonstar-shaped cases?

\section{BRIESKORN COMPLETE INTERSECTION SINGULARITIES}

In this section, we review some basic facts on the Brieskorn complete intersection (BCI for short) surface singularities and study arithmetic properties of invariants of those singularities. Then we show that a BCI singularity with $g \leq 1$ always has the maximal geometric genus and its maximal ideal cycle coincides with the fundamental cycle on the minimal good resolution. We basically use the notation of Section 2.

Recall that $\pi: X \rightarrow V$ denotes the minimal good resolution of a normal surface singularity $(V, o)$ with exceptional set $E$. 
3.1. The cycles and the Seifert invariants. We summarize the results in [12] which will be used in this section; those are a natural extension of the results on the hypersurface case obtained by Konno and Nagashima [8]. We assume that $(V, o)$ is a BCI normal surface singularity, namely, $V \subset \mathbb{C}^{m}$ can be defined as

$$
V=\left\{\left(x_{i}\right) \in \mathbb{C}^{m} \mid q_{i 1} x_{1}^{a_{1}}+\cdots+q_{i m} x_{m}^{a_{m}}=0, \quad i=3, \ldots, m\right\},
$$

where $a_{i}$ are integers such that $2 \leq a_{1} \leq \cdots \leq a_{m}$ and $q_{i j} \in \mathbb{C}$.

We define positive integers $\ell, \ell_{i}, \alpha, \alpha_{i}, \beta_{i}, \hat{g}, \hat{g}_{i}$, and $e_{i}$ as follows: ${ }^{2}$

$$
\begin{gathered}
\ell:=\operatorname{lcm}\left\{a_{1}, \ldots, a_{m}\right\}, \quad \ell_{i}:=\operatorname{lcm}\left(\left\{a_{1}, \ldots, a_{m}\right\} \backslash\left\{a_{i}\right\}\right), \\
\alpha_{i}:=\ell / \ell_{i}, \quad \alpha:=\alpha_{1} \cdots \alpha_{m}, \quad \hat{g}:=a_{1} \cdots a_{m} / \ell, \quad \hat{g}_{i}:=\hat{g} \alpha_{i} / a_{i}, \quad e_{i}:=\ell / a_{i}, \\
e_{i} \beta_{i}+1 \equiv 0 \quad\left(\bmod \alpha_{i}\right) \text { and } 0 \leq \beta_{i}<\alpha_{i} .
\end{gathered}
$$

We easily see that the polynomials appeared in (3.1) are weighted homogeneous polynomials of degree $\ell$ with respect to the weights $\left(e_{1}, \ldots, e_{m}\right)$ and that $\operatorname{gcd}\left\{\alpha_{i}, \alpha_{j}\right\}=$ 1 for $i \neq j$.

Definition 3.1. Let $Z^{(i)}=\left(x_{i}\right)_{E}$, the exceptional part of the $\operatorname{divisor}_{\operatorname{div}}{ }_{X}\left(x_{i}\right)$.

The next result follows from Theorem 4.4, 5.1, 6.1 of [12].

Theorem 3.2. We have the following.

(1) The resolution graph of $(V, o)$ is as in Figure $3\left(s_{i}=0\right.$ if $\left.\alpha_{i}=1\right)$, where

$$
E=E_{0}+\sum_{i=1}^{m} \sum_{\nu=1}^{s_{i}} \sum_{\xi=1}^{\hat{g}_{i}} E_{i, \nu, \xi}
$$

and the Seifert invariant is given by the following:

$$
\begin{gathered}
2 g-2=(m-2) \hat{g}-\sum_{i=1}^{m} \hat{g}_{i}, \\
c_{0}=\sum_{i=1}^{m} \frac{\hat{g}_{i} \beta_{i}}{\alpha_{i}}+\frac{a_{1} \cdots a_{m}}{\ell^{2}}, \quad \beta_{i} / \alpha_{i}= \begin{cases}{\left[\left[c_{i, 1}, \ldots, c_{i, s_{i}}\right]\right]^{-1}} & \text { if } \alpha_{i} \geq 2 \\
0 & \text { if } \alpha_{i}=1 .\end{cases}
\end{gathered}
$$

(2) For $1 \leq i \leq m$, we have

$$
\operatorname{cff}_{E_{0}}\left(Z^{(i)}\right)=e_{i}=\operatorname{deg}\left(x_{i}\right), \quad Z^{(i)}= \begin{cases}\sum_{\xi=1}^{\hat{g}_{i}} E_{i, s_{i}, \xi}^{*} & \text { if } \alpha_{i} \geq 2 \\ \hat{g}_{i} E_{0}^{*} & \text { if } \alpha_{i}=1 .\end{cases}
$$

Hence $Z^{(i)}=L_{e_{i}}$ for $1 \leq i \leq m$, and $M_{X}=Z^{(m)}$ since $e_{1} \geq \cdots \geq e_{m}$.

(3) We have $\operatorname{cff}_{E_{0}}\left(Z_{X}\right)=\min \left\{e_{m}, \alpha\right\}$ (cf. Remark 2.7) and

$$
Z_{X}= \begin{cases}M_{X} & \text { if } e_{m} \leq \alpha \\ \operatorname{deg}(\alpha D) E_{0}^{*} & \text { if } e_{m}>\alpha\end{cases}
$$

In particular, $Z_{X}=M_{X}$ if and only if $e_{m} \leq \alpha$.

Definition 3.3. We denote the weighted dual graph of Figure 3 by $\Gamma\left(a_{1}, \ldots, a_{m}\right)$.

Remark 3.4. We describe more precisely the situation of Theorem 3.2 (2). Let $H_{i}:=\operatorname{div}_{X}\left(x_{i}\right)-Z^{(i)}$. Then we have the decomposition $H_{i}=\bigcup_{\xi=1}^{\hat{g}_{i}} H_{i, \xi}$ into irreducible components such that

\footnotetext{
${ }^{2}$ Using the notation of $[12, \S 3]$, we have $l=d_{m}, \ell_{i}=d_{i m}, \alpha_{i}=n_{i m}, \beta_{i}=\mu_{i m}, e_{i}=e_{i m}$.
} 


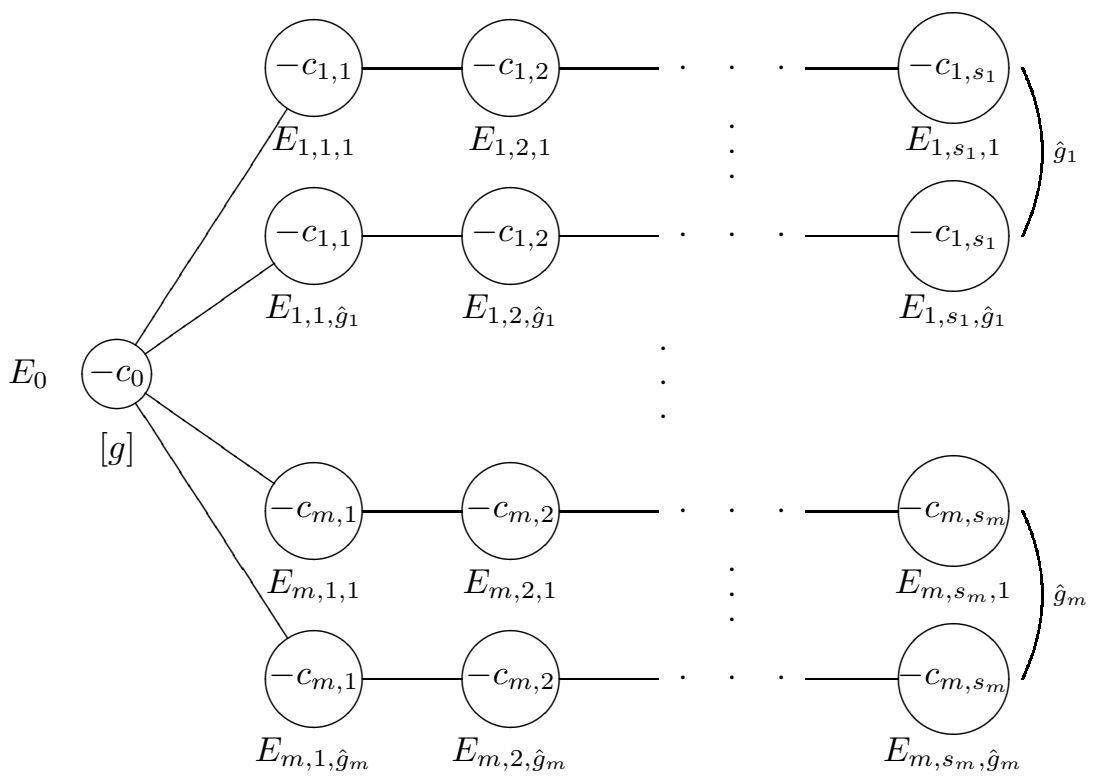

FIGURE 3 . The graph $\Gamma\left(a_{1}, \ldots, a_{m}\right)$

- $H_{i, \xi} E=H_{i, \xi} E_{i, s_{i}, \xi}=1$ if $\alpha_{i} \neq 1$,

- $H_{i, \xi} E=H_{i, \xi} E_{0}=1$ and $H_{i, \xi} \cap H_{i, \xi^{\prime}}=\emptyset\left(\xi \neq \xi^{\prime}\right)$ if $\alpha_{i}=1$.

In any cases, $H_{i} \cap H_{j}=\emptyset$ for $i \neq j$.

For $1 \leq i \leq m$, let $\left\{P_{i \xi} \mid \xi=1, \ldots, \hat{g}_{i}\right\} \subset E_{0}$ denote the set of points determined by $x_{i}=0$ in the weighted projective space $\mathbb{P}\left(e_{1}, \ldots, e_{m}\right)$. Then

$$
\left\{P_{i \xi}\right\}= \begin{cases}E_{0} \cap E_{i, 1, \xi} & \text { if } \alpha_{i} \neq 1, \\ E_{0} \cap H_{i, \xi} & \text { if } \alpha_{i}=1 .\end{cases}
$$

Let us recall that $\mathcal{O}_{E_{0}}\left(-L_{n}\right) \cong \mathcal{O}_{E_{0}}\left(D_{n}\right)$ (see Theorem 2.6) and $D_{\alpha}=\alpha D$.

Lemma 3.5. We have the following.

(1) For $n \in \mathbb{Z}_{>0}, \alpha \mid n$ if and only if $L_{n}=\left(\operatorname{deg} D_{n}\right) E_{0}^{*}$. In particular, if $\operatorname{deg} D_{e_{i}}>0$, then $\alpha \mid e_{i}$.

(2) If $d \in \mathbb{Z}_{>0}$ and $d E_{0}^{*} \in \mathbb{Z} E$, then $d E_{0}^{*}=L_{n}$, where $n=d \alpha / \operatorname{deg} D_{\alpha}$.

Proof. (1) Let $\phi: X \rightarrow X^{\prime}$ be the blowing-down of the divisor $E-E_{0}$. Then, at each point $\phi\left(P_{i \xi}\right) \in X^{\prime}\left(1 \leq i \leq m, 1 \leq \xi \leq \hat{g}_{i}\right)$, the reduced divisor $\phi\left(E_{0}\right)$ is a $\mathbb{Q}$-Cartier divisor and the order of $\left[\phi\left(E_{0}\right)\right] \in \mathrm{Cl}\left(X^{\prime}, \phi\left(P_{i \xi}\right)\right)$ is $\alpha_{i}$ (see Section 2.1). As in [13, II (b)], we have the pull-back $\phi^{*} \phi\left(E_{0}\right)$. Then $E_{0}^{*}=\operatorname{cff}_{E_{0}}\left(E_{0}^{*}\right)\left(\phi^{*} \phi\left(E_{0}\right)\right)$. Since $\alpha_{i}$ 's are pairwise relatively prime, $\alpha$ is the minimal positive integer such that $\alpha \phi\left(E_{0}\right)$ is a Cartier divisor on $X^{\prime}$, or equivalently, $\phi^{*}\left(\alpha \phi\left(E_{0}\right)\right) \in \mathbb{Z} E$. Hence $\alpha \mid n$ if and only if $\phi^{*}\left(n \phi\left(E_{0}\right)\right) \in \mathbb{Z} E$. If this is the case, $\phi^{*}\left(n \phi\left(E_{0}\right)\right)=L_{n}$ by Lemma $2.2(2)$, and moreover, $L_{n}=\left(-L_{n} E_{0}\right) E_{0}^{*}=\left(\operatorname{deg} D_{n}\right) E_{0}^{*}$. By Theorem 3.2 (2), $L_{e_{i}}=\left(\operatorname{deg} D_{e_{i}}\right) E_{0}^{*}$ if $\operatorname{deg} D_{e_{i}}>0$.

(2) As seen above, $d E_{0}^{*}=L_{n}$ by Lemma $2.2(2)$. Then $n=d \mathrm{cff}_{E_{0}}\left(E_{0}^{*}\right)$. From (1), we have $\alpha=\operatorname{deg} D_{\alpha} \operatorname{cff}_{E_{0}}\left(E_{0}^{*}\right)$. 
3.2. The coordinate ring and the semigroups. By virtue of Theorem 3.2 , we can write down the Pinkham-Demazure divisor as follows:

$$
D=Q-\Delta, \quad \Delta=\sum_{i=1}^{m} \frac{\beta_{i}}{\alpha_{i}} \bar{P}_{i}, \quad \bar{P}_{i}=\sum_{\xi=1}^{\hat{g}_{i}} P_{i \xi} \quad\left(\beta_{i}=0 \text { if } \alpha_{i}=1\right) .
$$

Definition 3.6. We call a cycle $C \geq 0$ a monomial cycle if $C=\sum_{i=1}^{m} m_{i} Z^{(i)}$ with $m_{i} \in \mathbb{Z}_{\geq 0}$, and write $x(C)=\prod_{i=1}^{m} x_{i}^{m_{i}}$. Clearly, $(x(C))_{E}=C$.

Remark 3.7. Let $C>0$ be an anti-nef $\mathbb{Q}$-cycle. Suppose that $\alpha_{i}>1$ for $i \leq s$ and $\alpha_{i}=1$ for $i>s$. If, for each $i \leq s, c_{i}:=C E_{i, s_{i}, \xi}$ is non-negative integer independent of $1 \leq \xi \leq \hat{g}_{i}$, and if the intersection numbers of $C$ and the exceptional components other than $E_{i, s_{i}, \xi}\left(i \leq s, 1 \leq \xi \leq \hat{g}_{i}\right)$ are zero, then $C$ is a monomial cycle since $C=\sum_{i=1}^{s} c_{i} Z^{(i)}$.

On the other hand, even if $C \in \mathbb{Z} E$ and $C=c E_{0}^{*}$ for some $c \in \mathbb{Z}_{>0}, C$ is not necessarily a monomial cycle. For example, if $\alpha<e_{m}$, then $L_{\alpha}=\left(\operatorname{deg} D_{\alpha}\right) E_{0}^{*}$ is not a monomial cycle (see Lemma 3.5, Theorem $3.2(2)$ ).

Let $\left\langle m_{1}, \ldots, m_{k}\right\rangle \subset \mathbb{Z}_{\geq 0}$ denote the numerical semigroup generated by integers $m_{1}, \ldots, m_{k} \in \mathbb{Z}_{\geq 0}$. For $n \in \mathbb{Z}_{\geq 0}$, let $R_{n}=H^{0}\left(D_{n}\right) T^{n} \subset R:=R(V, o)$, the vector space of homogeneous functions of degree $n$ (see Section 2.2).

Proposition 3.8. Let $n \in \mathbb{Z}_{\geq 0}$. We have the following.

(1) If $\operatorname{deg} D_{n} \in\left\langle\hat{g}_{1}, \ldots, \hat{g}_{m}\right\rangle$, then there exists a monomial cycle $W$ such that $\operatorname{cff}_{E_{0}}(W)=n$, and hence $h^{0}\left(D_{n}\right) \neq 0$.

(2) If $\operatorname{deg} D_{n}=\operatorname{deg} D_{k} \in\left\langle\hat{g}_{1}, \ldots, \hat{g}_{m}\right\rangle$ for some $k \geq 0$, then $D_{n} \sim D_{k}$. In particular, if $\operatorname{deg} D_{n}=0$, then $D_{n} \sim 0$.

(3) If $d:=\operatorname{deg} D_{n}>0$, then $d E_{0}^{*} \in \mathbb{Z} E$ and $\operatorname{deg} D_{\alpha} \mid d$.

Proof. (1) We first assume that $\operatorname{deg} D_{n}=0$. If $\alpha_{i}>1$, then $\operatorname{cff}_{E_{i, j, \xi}}\left(L_{n}\right)$ is independent of $1 \leq \xi \leq \hat{g}_{i}$ for each $1 \leq j \leq s_{i}$ (see Figure 3 ). Therefore, by Lemma 2.2 (1), there exists a cycle $F>0$ such that $L:=L_{n}+F$ is a monomial cycle with $\operatorname{cff}_{E_{0}}(L)=\operatorname{cff}_{E_{0}}\left(L_{n}\right)=n$ and $L E_{0}=0$ (cf. Remark 3.7). Then $x(L) \in R_{n}$.

Next assume that $\operatorname{deg} D_{n}=c_{1} \hat{g}_{1}+\cdots+c_{m} \hat{g}_{m}>0\left(c_{i} \in \mathbb{Z}_{\geq 0}\right)$. We may assume that $\alpha_{i}>1$ for $i \leq s$ and $\alpha_{i}=1$ for $i>s$. For $i \leq s$, let $F_{i}=\sum_{\xi=1}^{\hat{g}_{i}} \sum_{j=1}^{s_{i}} E_{i, j, \xi}$. Since $F_{i}$ is anti-nef on its support and $\operatorname{deg} D_{n}=-L_{n} E_{0}$, it follows from Theorem 3.2 (2) that the cycle

$$
W^{\prime}=L_{n}+\sum_{i=1}^{s} c_{i} F_{i}-\sum_{i=s+1}^{m} c_{i} Z^{(i)}
$$

is anti-nef and $W^{\prime} E_{0}=0$. Applying the argument above to the cycle $W^{\prime}$, there exists a cycle $F^{\prime}>0$ such that $W^{\prime}+F^{\prime}$ is a monomial cycle with cff $_{E_{0}}\left(W^{\prime}\right)=$ $\operatorname{cff}_{E_{0}}\left(W^{\prime}+F^{\prime}\right)$ and $\left(W^{\prime}+F^{\prime}\right) E_{0}=0$. Hence

$$
W:=W^{\prime}+F^{\prime}+\sum_{i=s+1}^{m} c_{i} Z^{(i)}
$$

is also a monomial cycle and $\mathrm{cff}_{E_{0}}(W)=\operatorname{cff}_{E_{0}}\left(W^{\prime}+\sum_{i=s+1}^{m} c_{i} Z^{(i)}\right)=n$. Thus, we obtain that $x(W) \in R_{n}$.

(2) We denote by $C_{n}$ the monomial cycle $W^{\prime}+F^{\prime}$ above, and also by $C_{k}$ the monomial cycle obtained from $L_{k}$ in the same manner as above. Since $C_{n}-C_{k}=$ 
$L_{n}-L_{k}$, on a suitably small neighborhood of $E_{0} \subset X$, we have

$$
L_{n}-L_{k}=\operatorname{div}_{X}\left(x\left(C_{n}\right) / x\left(C_{k}\right)\right) \sim 0 .
$$

Hence $D_{n}-\left.D_{k} \sim\left(-L_{n}+L_{k}\right)\right|_{E_{0}} \sim 0$.

(3) Since $\operatorname{deg} D_{n}=-L_{n} E_{0}, L_{n}-d E_{0}^{*}$ is an anti-nef $\mathbb{Q}$-cycle with $\left(L_{n}-d E_{0}\right) E_{0}=$ 0 . By the argument above, there exists a cycle $F>0$ such that $L_{n}-d E_{0}^{*}+F$ is a monomial cycle. Hence $d E_{0}^{*}$ is also a cycle (cf. Remark 3.7). We have $\operatorname{deg} D_{\alpha} \mid d$ by Lemma 3.5.

Theorem 3.9. If $g \leq 1$, then $p_{g}(V, o)=p_{g}\left(\Gamma\left(a_{1}, \ldots, a_{m}\right)\right)$ (see Definition 2.8).

Proof. By Pinkham's formula, $p_{g}(V, o)=\sum_{n \geq 0} h^{1}\left(D_{n}\right)$. If $g=0$, then this is topological, and the assertion is clear. Suppose that $g=1$. If $\operatorname{deg} D_{n} \neq 0$, then $h^{1}\left(D_{n}\right)$ is topological by Riemann-Roch theorem and Serre duality, namely, independent of the complex structure of $(V, o)$. If $\operatorname{deg} D_{n}=0$, then $h^{1}\left(D_{n}\right)=h^{0}\left(D_{n}\right)=1$ by Proposition 3.8. Hence $p_{g}(V, o)=p_{g}\left(\Gamma\left(a_{1}, \ldots, a_{m}\right)\right)$.

Theorem 3.10. We have the following.

(1) $\left\langle e_{1}, \ldots, e_{m}\right\rangle=\left\{n \in \mathbb{Z}_{\geq 0} \mid h^{0}\left(D_{n}\right) \neq 0\right\}$.

(2) For $n \in \mathbb{Z}_{\geq 0}, n \in\left\langle e_{1}, \ldots, e_{m}\right\rangle$ if and only if $\operatorname{deg} D_{n} \in\left\langle\hat{g}_{1}, \ldots, \hat{g}_{m}\right\rangle$.

Proof. (1) follows from the fact that $R=\bigoplus_{k \geq 0} H^{0}\left(D_{k}\right) T^{k}$ is generated by the elements $x_{1}, \ldots, x_{m}$ with $\operatorname{deg} x_{i}=e_{i}$.

(2) The "if" part follows from Proposition 3.8 (1). Assume that $n=\sum_{i=1}^{m} m_{i} e_{i}$ with $m_{i} \geq 0$. Then the monomial cycle $M:=\sum_{i=1}^{m} m_{i} Z^{(i)}$ satisfies $\operatorname{cff}_{E_{0}}(M)=n$. We proceed in a similar way as in the proof of Proposition 3.8. We may assume that $\alpha_{i}>1$ for $i \leq s$ and $\alpha_{i}=1$ for $i>s$. Then $-M E_{0}=\sum_{i>s} m_{i} \hat{g}_{i} \in\left\langle\hat{g}_{1}, \ldots, \hat{g}_{m}\right\rangle$ by Theorem $3.2(2)$. Let $W=M-\sum_{i>s} m_{i} Z^{(i)}$ and $n^{\prime}=\operatorname{cff}_{E_{0}}(W)$. Clearly, $W$ is also a monomial cycle. By the definition of $L_{n^{\prime}}$, we have $\operatorname{cff}_{E_{0}}\left(W-L_{n^{\prime}}\right)=0$ and $W-L_{n^{\prime}} \geq 0$. Since $\operatorname{cff}_{E_{i, j, \xi}}\left(L_{n^{\prime}}\right)$ and $\left.\mathrm{cff}_{E_{i, j, \xi}}(W)\right)$ are independent of $1 \leq \xi \leq \hat{g}_{i}$ for each $1 \leq j \leq s_{i}$, we obtain that $\left(W-L_{n^{\prime}}\right) E_{0} \in\left\langle\hat{g}_{1}, \ldots, \hat{g}_{m}\right\rangle$. On the other hand, $L_{n}=L_{n^{\prime}}+(M-W)$ by Lemma $2.2(4)$. Therefore,

$$
\operatorname{deg} D_{n}=-L_{n} E_{0}=\left(W-L_{n^{\prime}}-M\right) E_{0} \in\left\langle\hat{g}_{1}, \ldots, \hat{g}_{m}\right\rangle .
$$

Corollary 3.11. If $g>0$, then $a(R) \in\left\langle e_{1}, \ldots, e_{m}\right\rangle$ and $2 g-2 \in\left\langle\hat{g}_{1}, \ldots, \hat{g}_{m}\right\rangle$. Note that $a(R)=(m-2) \ell-\sum_{i=1}^{m} e_{i}$ by $[6,3.1 .6]$.

Proof. By Theorem 2.5, $K_{E_{0}} \sim D_{a(R)}$. Since $h^{0}\left(K_{E_{0}}\right)=g>0$, the assertion follows from Theorem 3.10.

Theorem 3.12. If $H^{0}\left(D_{\alpha}\right) \neq 0$, then $M_{X}=Z_{X}$. In particular, if $g \leq 1$, then $M_{X}=Z_{X}$.

Proof. If $H^{0}\left(D_{\alpha}\right) \neq 0$, then $\alpha \in\left\langle e_{1}, \ldots, e_{m}\right\rangle$ by Theorem 3.10. Hence $e_{m} \leq \alpha$, and $M_{X}=Z_{X}$ by Theorem 3.2. If $g \leq 1$, we have $H^{0}(D) \neq 0$ for any divisor $D$ on $E_{0}$ with $\operatorname{deg} D>0$.

Example 3.13. We have seen that if $\alpha<e_{m}$, then $H^{0}\left(D_{\alpha}\right)=0$ even though $D_{\alpha}>0$. We show that the condition $e_{m}<\alpha$ does not imply $H^{0}\left(D_{\alpha}\right) \neq 0$; thus, the converse of Theorem 3.12 does not hold.

Suppose that $\left(a_{1}, a_{2}, a_{3}\right)=(6,10,45)$. Then we have

$$
\left\{e_{1}, e_{2}, e_{3}\right\}=\{15,9,2\}, \quad\left\{\hat{g}_{1}, \hat{g}_{2}, \hat{g}_{3}\right\}=\{5,3,2\}, \quad \alpha=3, \quad \operatorname{deg} D_{\alpha}=1,
$$


and $H^{0}\left(D_{\alpha}\right)=0$ by Theorem 3.10. Note that the Seifert invariant is $(11,1,2(3,1))$. This is a hyperelliptic type (see Example 2.9). Hence $p_{g}(V, o)=p_{g}(\Gamma(6,10,45)$ ).

3.3. Non-BCI singularities. In the rest of this section, we assume that $(V, o)$ is an arbitrary weighted homogeneous singularity with resolution graph $\Gamma\left(a_{1}, \ldots, a_{m}\right)$. We use the same notation as above. Recall that the Pinkham-Demazure divisor is expressed as $D=Q-\Delta$.

Lemma 3.14. Assume that $\alpha \leq e_{m}$. Then $M_{X}=Z_{X}$ if and only if there exists an effective divisor $F$ on $E_{0}$ such that $\alpha D=D_{\alpha} \sim F$ and $\operatorname{Supp} F \cap \operatorname{Supp} \Delta=\emptyset$.

Proof. Let $c=\operatorname{deg} D_{\alpha}$. Since $\alpha \leq e_{m}$, it follows from Theorem 3.2 and Lemma 3.5 that $Z_{X}=L_{\alpha}=c E_{0}^{*}$ (note that the fundamental cycle is determined by the resolution graph). On the other hand, $M_{X}=Z_{X}$ if and only if there exists a function $h \in H^{0}\left(\mathcal{O}_{X}\left(-Z_{X}\right)\right)$ such that $\operatorname{div}_{X}(h)=Z_{X}+H$, where $H$ is the nonexceptional part. In this case, we have $E H=E_{0} H$ since $H \sim-c E_{0}^{*}$. Thus $\left(E-E_{0}\right) H=0$. Let $F=\left.H\right|_{E_{0}}$. Then $\operatorname{Supp} F \cap \operatorname{Supp} \Delta=\emptyset$ and $D_{\alpha} \sim-\left.L_{\alpha}\right|_{E_{0}} \sim F$.

Conversely, suppose that $D_{\alpha} \sim F>0$ and $\operatorname{Supp} F \cap \operatorname{Supp} \Delta=\emptyset$. Since $H^{0}\left(D_{\alpha}\right) \neq 0$, there exists $h \in H^{0}\left(\mathcal{O}_{X}\right)$ such that $\operatorname{div}_{X}(h)=c E_{0}^{*}+E^{\prime}+H$ where $E^{\prime}$ is a cycle supported in $E-E_{0}$ and $H$ is the non-exceptional part. By assumption, $\left.\left(E^{\prime}+H\right)\right|_{E_{0}} \sim-\left.L_{\alpha}\right|_{E_{0}} \sim F$. In fact, we may assume that $\left.\left(E^{\prime}+H\right)\right|_{E_{0}}=F$, since the restriction map $H^{0}\left(\mathcal{O}_{X}\left(-L_{n}\right)\right) \rightarrow H^{0}\left(\mathcal{O}_{E_{0}}\left(D_{n}\right)\right)$ is surjective by Theorem 2.6. Then $\left.H\right|_{E_{0}}=F$ by the assumption on the supports, and $E^{\prime}=0$ since $E^{\prime 2}=\operatorname{div}_{X}(h) E^{\prime}=0$.

Lemma 3.15. For any effective divisor $F \in \operatorname{Div}\left(E_{0}\right)$ such that $\operatorname{deg} F=\operatorname{deg} \alpha D$, there exists a divisor $\widetilde{Q} \in \operatorname{Div}\left(E_{0}\right)$ such that

$$
F \sim \alpha \widetilde{Q}-\alpha \Delta .
$$

Let $\widetilde{D}=\widetilde{Q}-\Delta$ and $\widetilde{R}=R\left(E_{0}, \widetilde{D}\right)$ (see Section 2.3). If $R=R\left(E_{0}, D\right)$ is a Gorenstein ring, then $\widetilde{R}$ is also Gorenstein if and only if $a(\widetilde{Q}-Q) \sim 0$, where $a=a(R)$.

Proof. Since $\operatorname{deg}(F-\alpha D)=0$, there exists a divisor $Q_{F}$ with $\operatorname{deg} Q_{F}=0$ such that $\alpha Q_{F} \sim F-\alpha D$. Let $\widetilde{Q}=Q_{F}+Q$. Then

$$
\alpha \widetilde{Q}-\alpha \Delta \sim \alpha Q_{F}+\alpha Q-\alpha \Delta \sim F .
$$

Let $D^{\prime}$ be the $\mathbb{Q}$-divisor as in Theorem 2.5, and assume that $R$ is Gorenstein. Then $K_{E_{0}} \sim a D-D^{\prime}$, and $\widetilde{R}$ is Gorenstein if and only if $\left(a D-D^{\prime}\right) \sim\left(a \widetilde{D}-D^{\prime}\right)$.

Theorem 3.16. There exists a weighted homogeneous singularity with resolution graph $\Gamma\left(a_{1}, \ldots, a_{m}\right)$ such that the maximal ideal cycle coincides with the fundamental cycle on the minimal good resolution.

Proof. Let $(V, o)$ be a BCI singularity. If $e_{m} \leq \alpha$, we have $M_{X}=Z_{X}$ by Theorem 3.2. If $e_{m}>\alpha$, by Lemma 3.14 and 3.15, we can take a Pinkham-Demazure divisor $\widetilde{D}$ on $E_{0}$ so that $\operatorname{Spec} R\left(E_{0}, \widetilde{D}\right)$ satisfies the assertion.

\section{Examples of Singularities in $\mathcal{X}(\Gamma(2,3,3,4))$}

We study some special structures of weighted homogeneous singularities with resolution graph $\Gamma(2,3,3,4)$. The tuple of integers $\left(a_{1}, a_{2}, a_{3}, a_{4}\right)=(2,3,3,4)$ is 
TOMOHIRO OKUMA

characterized by the properties that $a_{1}+\cdots+a_{m}\left(a_{i} \geq 2\right)$ is minimal such that the corresponding BCI singularity satisfies $E \neq E_{0}$ and $g=2$.

Let $\Gamma=\Gamma(2,3,3,4)$ and let $\overline{\mathcal{X}}(\Gamma)$ denote the set of weighted homogeneous singularities with resolution graph $\Gamma$. We shall show that the singularities in $\overline{\mathcal{X}}(\Gamma)$ which attain the maximal geometric genus $p_{g}(\Gamma)$ (see Definition 2.8) are of hyperelliptic type, and obtain the equations for them. Moreover, we classify the singularities in $\overline{\mathcal{X}}(\Gamma)$ with the property that the maximal ideal cycle coincides with the fundamental cycle.

In the following, we use the notation of Section 3. Notice that the coefficients of the cycles $Z_{X}, L_{n}$, and $Z_{K_{X}}$ are determined by $\Gamma$.

First, we give the fundamental invariants of BCI singularities with resolution graph $\Gamma$ (cf. Section 3.1); these data and the following theorem are used in other subsections.

Notation 4.1. Let $\operatorname{mult}(V, o)$ (resp. $\operatorname{embdim}(V, o)$ ) denote the multiplicity (resp. embedding dimension) of the singularity $(V, o)$, namely, that of the local ring $\mathcal{O}_{V, o}$.

Theorem 4.2. Let $A:=\mathcal{O}_{W, p}$ be the local ring of a d-dimensional Cohen-Macaulay complex space $W$ at $p \in W$. Then we have the following.

(1) (Abhyankar [1]) embdim $A \leq$ mult $A+d-1$.

(2) (Sally [25]) If $A$ is Gorenstein and mult $A \geq 3$, then embdim $A \leq$ mult $A+$ $d-2$.

(3) (Serre [26]) If $A$ is Gorenstein and $\operatorname{embdim} A=d+2$, then $A$ is a complete intersection.

4.1. The BCI singularities. Assume that $(V, o)$ is a BCI surface singularity with $\left(a_{1}, \ldots, a_{4}\right)=(2,3,3,4)$. Then $V$ can be defined by polynomials

$$
f_{1}:=x_{1}^{2}+x_{2}^{3}+p x_{3}^{3}, \quad f_{2}:=x_{2}^{3}+x_{3}^{3}+x_{4}^{4} \quad(p \neq 0,1) .
$$

These are weighted homogeneous of $\operatorname{deg} f_{i}=\ell=12$ with respect to the weights

$$
\left(\operatorname{deg} x_{1}, \ldots, \operatorname{deg} x_{4}\right)=\left(e_{1}, \ldots, e_{4}\right)=(6,4,4,3) .
$$

We also have $\left(\alpha_{1}, \ldots, \alpha_{4}\right)=(1,1,1,2)$. By $[12,6.3], \operatorname{mult}(V, o)=a_{1} a_{2}=6$. Let $R=\mathbb{C}\left[x_{1}, \ldots, x_{4}\right] /\left(f_{1}, f_{2}\right)$. It follows from $[6,3.1 .6]$ that

$$
a(R)=12+12-(6+4+4+3)=7 .
$$

The Hilbert series of $R$ is

$$
H(V, t)=\frac{\left(1-t^{12}\right)^{2}}{\left(1-t^{3}\right)\left(1-t^{4}\right)^{2}\left(1-t^{6}\right)}=1+t^{3}+2 t^{4}+2 t^{6}+2 t^{7}+3 t^{8}+\cdots .
$$

By Proposition 2.4 (1), we have

$$
p_{g}(V, o)=\left.\left(2+2 t+2 t^{3}+t^{4}+t^{7}\right)\right|_{t=1}=8 .
$$

From the result of Section 3.1, we have the resolution graph $\Gamma$ as Figure 4.

Since $\alpha=2<e_{4}$, we have $Z_{X} \neq M_{X}$ by Theorem 3.2. In fact, we have that

$$
Z_{X}=L_{2}=E+E_{0}=E_{0}^{*}, \quad M_{X}=Z^{(4)}=L_{3}=Z_{X}+E, \quad Z_{K_{X}}=4 Z_{X} .
$$

The fundamental genus is $p_{a}\left(Z_{X}\right)=h^{1}\left(\mathcal{O}_{Z_{X}}\right)=1+Z_{X}\left(Z_{X}+K_{X}\right) / 2=4$. The arithmetic genus of $(V, o)$ is defined by $p_{a}(V, o)=\max \left\{p_{a}(D) \mid D>0\right.$ is a cycle $\}$. It is known that $p_{a}\left(Z_{X}\right) \leq p_{a}(V, o) \leq p_{g}(V, o)$ (see [32]). By Koyama's inequality (see [7, Proposition 1.6]), we have $p_{a}(V, o)=p_{a}\left(2 Z_{X}\right)=5$. 


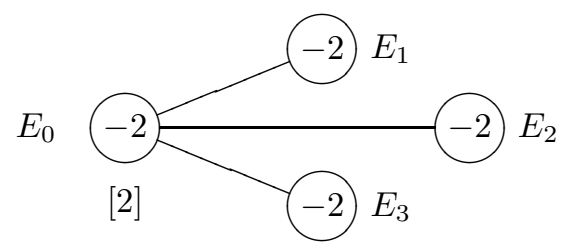

FiguRE $4 . \quad \Gamma=\Gamma(2,3,3,4)$

The Pinkham-Demazure divisor $D$ and $D_{n}$ are as follows:

$$
D=Q-\sum_{i=1}^{3} \frac{1}{2} P_{i}, \quad D_{n}=n Q-\sum_{i=1}^{3}\left\lceil\frac{n}{2}\right\rceil P_{i},
$$

where $\mathcal{O}_{E_{0}}(Q)=\mathcal{O}_{E_{0}}\left(-E_{0}\right)$ and $\left\{P_{i}\right\}=E_{0} \cap E_{i}$. Since $\operatorname{deg} Q=2$, we have the following table; these are topological invariant and also used in Section 4.2-4.3.

\begin{tabular}{c|c|c|c|c|c|c|c}
\hline$n$ & 1 & 2 & 3 & 4 & 5 & 6 & 7 \\
\hline $\operatorname{deg} D_{n}$ & -1 & 1 & 0 & 2 & 1 & 3 & 2 \\
\hline
\end{tabular}

The divisor $D$ satisfies the following analytic condition.

Lemma 4.3. $Q \sim 2 P_{i} \sim K_{E_{0}}$ for $i=1,2,3$.

Proof. Since $a(R)=7$, by Theorem 2.5 and Proposition 3.8 (2),

$$
K_{E_{0}} \sim D_{7} \sim D_{7}-2 D_{3}=Q .
$$

Note that $E_{0}$ is a hyperelliptic curve with $g=2$. From Remark 3.4, we see that $\left\{P_{1}, P_{2}, P_{3}\right\}=\left\{f_{1}=f_{2}=x_{4}=0\right\} \subset \mathbb{P}(6,4,4,3)$. Thus, a double cover $E_{0} \rightarrow \mathbb{P}^{1}$ is given by $\left(x_{1}: x_{2}: x_{3}: x_{4}\right) \mapsto\left(x_{2}: x_{3}\right)$ and $P_{i}$ are its ramification points. Hence $2 P_{i} \sim K_{E_{0}}$.

Later, we shall see the variation of the Pinkham-Demazure divisor $D$ and corresponding singularities with $\Gamma=\Gamma(2,3,3,4)$.

4.2. Singularities with $p_{g}=p_{g}(\Gamma)$. Let $C$ be a nonsingular curve of genus two and $\left\{P_{1}, P_{2}, P_{3}\right\} \subset C$ a set of distinct three points. Let $Q$ be a divisor on $C$ with $\operatorname{deg} Q=2$. We define $D$ and $D_{n}\left(n \in \mathbb{Z}_{\geq 0}\right)$ as in (4.2). Suppose that $(V, o) \in \overline{\mathcal{X}}(\Gamma)$ and the homogeneous coordinate ring $R$ of $(V, o)$ is expressed as $R=\bigoplus_{n \geq 0} H^{0}\left(D_{n}\right) T^{n}$, where $H^{0}\left(D_{n}\right)=H^{0}\left(C, \mathcal{O}_{C}\left(D_{n}\right)\right)$ (see Section 2.3). For $n \in \mathbb{Z}_{\geq 0}$, let $R_{n}=H^{0}\left(D_{n}\right) T^{n}$. We identify $C$ with the central curve $E_{0} \subset E$.

Lemma 4.4. The following are equivalent.

(1) $(V, o)$ is Gorenstein.

(2) $K_{C}$ is linearly equivalent to $D_{7}$.

(3) $h^{0}\left(D_{7}\right)=2$.

In this case, we have $a(R)=7$.

Proof. Since $g=g(C)=2$, for a divisor $F$ of degree 2 on $C, h^{0}(F)=2$ if and only if $F \sim K_{C}$. The assertion follows from Theorem 2.5.

Notation 4.5. Let $\mathcal{R}(C) \subset C$ be the set of ramification points of the double cover $C \rightarrow \mathbb{P}^{1}$ and $\sigma: C \rightarrow C$ the hyperelliptic involution; we have $\mathcal{R}(C)=$ $\{P \in C \mid \sigma(P)=P\}$. 
From Example 2.9, we have the following.

Proposition 4.6. Assume that $P_{1} \in \mathcal{R}(C), P_{2} \in C \backslash \mathcal{R}(C), P_{3}=\sigma\left(P_{2}\right)$ and $Q=2 P_{1}$. Then

$$
D_{n} \sim \begin{cases}\frac{n}{2} P_{1} & (n \text { is even }) \\ \frac{n-3}{2} P_{1} & (n \text { is odd })\end{cases}
$$

and $p_{g}(V, o)=p_{g}(\Gamma)$.

We can prove the converse of the above result.

Proposition 4.7. Assume that $p_{g}(V, o)=p_{g}(\Gamma)$. Then $D$ can be taken as in Proposition 4.6, namely, by suitable permutation of $P_{i}$ 's, we have $P_{1} \in \mathcal{R}(C)$, $P_{2} \in C \backslash \mathcal{R}(C), P_{3}=\sigma\left(P_{2}\right)$, and $Q \sim 2 P_{1}$.

Proof. By Proposition 2.4 (2) and Clifford's theorem (cf. Example 2.9), we have

$$
h^{0}\left(D_{n}\right)=\left\lfloor\operatorname{deg} D_{n} / 2\right\rfloor+1 \quad \text { if } \quad \operatorname{deg} D_{n} \leq 2 .
$$

Since $\operatorname{deg} D_{2}=1$ and $h^{0}\left(D_{2}\right)=1$, there exists a point $P_{4} \in C$ such that

$$
D_{2}=2 Q-\left(P_{1}+P_{2}+P_{3}\right) \sim P_{4} \text {. }
$$

Since $\operatorname{deg} D_{3}=0$ and $h^{0}\left(D_{3}\right)=1$, it follows that

$$
D_{3}=3 Q-2\left(P_{1}+P_{2}+P_{3}\right) \sim 0
$$

From (4.5) and (4.6), we have $D_{4} \sim 2 P_{4} \sim Q$. Since $h^{0}\left(D_{4}\right)=2$, we have $P_{4} \in$ $\mathcal{R}(C)$. Therefore, $P+\sigma(P) \sim Q$ for any $P \in C$. It follows from (4.5) that

$$
P_{1}+P_{2}+P_{3} \sim Q+P_{4} \sim P_{1}+\sigma\left(P_{1}\right)+P_{4} .
$$

Hence $P_{2}+P_{3} \sim \sigma\left(P_{1}\right)+P_{4}$. If $P_{2}+P_{3}=\sigma\left(P_{1}\right)+P_{4}$, we are done (e.g., if $P_{2}=P_{4}$, then $\left.P_{2} \in \mathcal{R}(C), \sigma\left(P_{1}\right)=P_{3} \notin \mathcal{R}(C)\right)$. If $P_{2}+P_{3} \neq \sigma\left(P_{1}\right)+P_{4}$, then $h^{0}\left(\sigma\left(P_{1}\right)+P_{4}\right)=2$, and this implies that $P_{1}=P_{4}$ and $P_{3}=\sigma\left(P_{2}\right)$.

We shall give the fundamental invariants of these singularities.

For an invertible sheaf $\mathcal{L}$ on $X$, we say that $P \in X$ is a base point of $\mathcal{L}$ if $\mathcal{L}$ is not generated by its global sections at $P$.

Lemma 4.8 (cf. [32, 2.7], [24, 4.6]). If $\mathcal{O}_{X}\left(-M_{X}\right)$ has no base points, then $\operatorname{mult}(V, o)=-M_{X}^{2}$.

Proposition 4.9. Assume that $p_{g}(V, o)=p_{g}(\Gamma)$. Then we have the following.

(1) $M_{X}=Z_{X}+E_{1}$, where $P_{1}$ is taken as in Proposition 4.6. Furthermore, $\mathcal{O}_{X}\left(-M_{X}\right)$ has no base points and $\operatorname{mult}(V, o)=4$.

(2) $p_{g}(V, o)=10$.

(3) $(V, o)$ is a complete intersection singularity defined as

$$
V=\left\{(x, y, z, w) \in \mathbb{C}^{4} \mid y^{2}-x z=w^{2}-h_{5}\left(x^{2}, z\right)=0\right\},
$$

where $h_{5}$ is a homogeneous polynomial of degree 5. This is a weighted homogeneous singularity of weight type $(2,3,4,10 ; 6,20)$.

Proof. Assume that $D$ is as in Proposition 4.6. It follows from Lemma 4.4 that $(V, o)$ is Gorenstein, because $K_{C} \sim 2 P_{1} \sim D_{7}$.

(1) Since $h^{0}\left(D_{2}\right)>0$, there exists a homogeneous function $h \in R_{2}$ such that $\operatorname{div}_{X}(h)=Z_{X}+F+H$, where $F$ is a cycle satisfying $0 \leq F \leq E_{1}+E_{2}+E_{3}$ and 
$H$ is the non-exceptional part. Note that any point of $H \cap E$ is in $E_{0} \backslash\left\{P_{1}, P_{2}, P_{3}\right\}$ or $\left(E_{1} \cup E_{2} \cup E_{3}\right) \backslash E_{0}$, because $h$ is homogeneous. Since

$$
\left.0 \sim \operatorname{div}_{X}(h)\right|_{E_{0}} \sim-D_{2}+\left.(F+H)\right|_{E_{0}} \sim-P_{1}+\left.(F+H)\right|_{E_{0}},
$$

we have $F \cap E_{0}=\left\{P_{1}\right\}$ and $H \cap E_{0}=\emptyset$; thus $F=E_{1}$ and $E \cap H \subset E_{1} \backslash E_{0}$. Since $\operatorname{cff}_{E_{1}}\left(L_{n}\right) \geq 2$ for all $n \geq 3$, we have that $M_{X}=Z_{X}+E_{1}$ and $\mathcal{O}_{X}\left(-M_{X}\right)$ is generated by global sections outside $E_{1} \cap H$. Since $L_{4}=2 E_{0}^{*}$ and $D_{4} \sim 2 P_{0}$ for any $P_{0} \in \mathcal{R}(C) \backslash\left\{P_{1}\right\}$, there exists $g \in R$ such that $\operatorname{div}_{X}(g)=L_{4}+H^{\prime}$ where $H^{\prime}$ intersects $E_{0}$ only at $P_{0}$ (cf. the proof of Lemma 3.14). Since $\mathrm{cff}_{E_{1}}\left(M_{X}\right)=$ $\operatorname{cff}_{E_{1}}\left(L_{4}\right)=2$ and $L_{4} E_{1}=0, \mathcal{O}_{X}\left(-M_{X}\right)$ has no base points. Hence $\operatorname{mult}(V, o)=$ $-\left(M_{X}\right)^{2}=4$ by Lemma 4.8 .

(2) Let $\left(V_{0}, o\right) \in \overline{\mathcal{X}}(\Gamma)$ be a BCI singularity. Since $\operatorname{deg} D_{n} \geq 3$ for $n \geq 8, h^{0}\left(D_{n}\right)$ with $n \geq 8$ is independent of the complex structure of the pair $(C, D)$. By (4.1) and (4.4), we have the Hilbert series $H(V, t)$ of $R=R(V, o)$ :

$$
\begin{aligned}
H(V, t) & =H\left(V_{0}, t\right)+t^{2}+t^{5}=\frac{\left(1-t^{6}\right)\left(1-t^{20}\right)}{\left(1-t^{2}\right)\left(1-t^{3}\right)\left(1-t^{4}\right)\left(1-t^{10}\right)} \\
& =1+t^{2}+t^{3}+2 t^{4}+t^{5}+2 t^{6}+2 t^{7}+3 t^{8}+2 t^{9}+4 t^{10}+\cdots .
\end{aligned}
$$

By Proposition $2.4(2), p_{g}(V, o)=p_{g}\left(V_{0}, o\right)+2=10$.

(3) From (4.7), we have the following functions belong to a minimal set of homogeneous generators of $\mathbb{C}$-algebra $R$ :

$$
x=f_{2} T^{2} \in R_{2}, \quad y=f_{3} T^{3} \in R_{3}, \quad z=f_{4} T^{4} \in R_{4}
$$

such that $\operatorname{div}_{E_{0}}\left(f_{i}\right) \geq D_{i}$. Since $x^{3}, y^{2}, x z \in H^{0}\left(D_{6}\right) T^{6}$ and $h^{0}\left(D_{6}\right)=2$, we have a relation $r_{6}(x, y, z)=0$ at degree 6 . Let $\mathbb{C}[X, Y, Z]$ be the polynomial ring with $(\operatorname{deg} X, \operatorname{deg} Y, \operatorname{deg} Z)=(2,3,4)$. The difference between the Hilbert series of $R$ and that of the quotient ring $\mathbb{C}[X, Y, Z] /\left(r_{6}(X, Y, Z)\right)$ is

$$
H(V, t)-\frac{\left(1-t^{6}\right)}{\left(1-t^{2}\right)\left(1-t^{3}\right)\left(1-t^{4}\right)}=t^{10}+\cdots .
$$

Hence we have an element $w \in R_{10}$ such that $\{x, y, z, w\}$ is a subset of a minimal set of homogeneous generators of $R$. However, since $(V, o)$ is Gorenstein and $\operatorname{mult}(V, o)=4$, it follows from Theorem 4.2 that $R$ is a complete intersection generated by just $x, y, z, w$ as $\mathbb{C}$-algebra. Let $F(t)$ be the Hilbert series of $\mathbb{C}[X, Y, Z, W] /\left(r_{6}(X, Y, Z)\right)$, where $\operatorname{deg} W=10$. Then

$$
H(V, t)-F(t)=-t^{20}+\cdots .
$$

Hence we have a relation $r_{20}(x, y, z, w)=0$ at degree 20 . Then the natural $\mathbb{C}$ homomorphism

$$
S:=\mathbb{C}[X, Y, Z, W] /\left(r_{6}(X, Y, Z), r_{20}(X, Y, Z, W)\right) \rightarrow R
$$

induced by $(X, Y, Z, W) \mapsto(x, y, z, w)$ is surjective and the Hilbert series of $S$ coincides with $H(V, t)$. Hence $S \cong R$.

Next we consider the equations. Suppose that $\phi: E_{0} \rightarrow \mathbb{P}^{1}$ is a double cover such that $\phi\left(P_{1}\right)=\left\{x_{0}=0\right\}$ and $\phi\left(P_{i}\right)=\left\{x_{1}=0\right\}(i=2,3)$, where $x_{0}$ and $x_{1}$ are the homogeneous coordinates of $\mathbb{P}^{1}$. Then $E_{0}$ can be defined by the equation $x_{2}^{2}=$ $x_{0} h_{5}\left(x_{0}, x_{1}\right)$, where $h_{5}\left(x_{0}, x_{1}\right)$ is a homogeneous polynomial of degree 5 such that $h_{5}(1,0) h_{5}(0,1) \neq 0$; the branch locus of the covering is $\left\{x_{0} h_{5}\left(x_{0}, x_{1}\right)=0\right\} \subset \mathbb{P}^{1}$. 
Now, we can put $x=x_{0} x_{1}, y=x_{0} x_{1}^{2}, z=x_{0} x_{1}^{3}, w=x_{0}^{2} x_{1}^{5} x_{2}$. Then we have the relations

$$
y^{2}=x_{0}^{2} x_{1}^{4}=x z, \quad w^{2}=h_{5}\left(x_{0}, x_{1}\right)\left(x_{0} x_{1}^{2}\right)^{5}=h_{5}\left(x^{2}, z\right) .
$$

4.3. Singularities with $M_{X}=Z_{X}$. We classify the singularities $(V, o) \in \overline{\mathcal{X}}(\Gamma)$ with property that $M_{X}=Z_{X}$. We use the notation of the preceding subsection.

Proposition 4.10. We have the following.

(1) $M_{X}=Z_{X}$ if and only if there exists a point $P_{4} \in C \backslash\left\{P_{1}, P_{2}, P_{3}\right\}$ such that $D_{2} \sim P_{4}$; if this is the case, $D_{7} \sim 4 P_{4}-Q$.

(2) Assume that $M_{X}=Z_{X}$ and that $x \in R_{2}$ and $y \in R_{m}$ belong to a minimal set of homogeneous generators of the $\mathbb{C}$-algebra $R$, where $m$ is the minimum of the degrees of those generators except for $x$. If $P_{4}$ is not a base point of $H^{0}\left(D_{m}\right)$, then $\operatorname{mult}(V, o)=m$.

Proof. (1) The equivalence follows from Lemma 3.14.

(2) We have $\operatorname{div}_{X}(x)=Z_{X}+H$, where $H$ is the non-exceptional part. Since $H \cap E=\left\{P_{4}\right\}, \mathcal{O}_{X}\left(-Z_{X}\right)$ has just a base point $P_{4}$. Assume that $u, v$ are the local coordinates at $P_{4} \in X$ such that $E_{0}=\{u=0\}$ and $H=\{v=0\}$. By the assumption, we may also assume that $x=u^{2} v$ and $y=u^{m}$. Note that $m \geq 3$ since $h^{0}\left(D_{2}\right)=1$. Then, at $P_{4} \in X, \mathfrak{m} \mathcal{O}_{X}=\left(u^{2} v, u^{m}\right) \mathcal{O}_{X}=\left(v, u^{m-2}\right) \mathcal{O}_{X}\left(-Z_{X}\right)$, where $\mathfrak{m} \subset \mathcal{O}_{V, o}$ is the maximal ideal. Therefore, the base point of $\mathcal{O}_{X}\left(-Z_{X}\right)$ is resolved by the composition $Y \rightarrow X$ of $m-2$ blowing-ups at the intersection of the exceptional set and the proper transform of $H$. Then the maximal ideal cycle $M_{Y}$ on $Y$ is the exceptional part of $\operatorname{div}_{Y}(x)$ and by Lemma $4.8, \operatorname{mult}(V, o)=-M_{Y}^{2}=$ $-Z_{X}^{2}+(m-2)=m$.

Remark 4.11. The proof of Proposition 4.10 shows that $\operatorname{mult}(W, o) \geq-Z_{X}^{2}+1=3$ for any normal surface singularity $(W, o)$ with resolution graph $\Gamma$.

Lemma 4.12. Let $P \in C$.

(1) $P \notin \mathcal{R}(C)$ if and only if the linear system $|3 P|$ is free.

(2) There exist distinct three points $A_{1}, A_{2}, A_{3} \in C$ such that $3 P \sim \sum_{i=1}^{3} A_{i}$. For such points, $P \in \mathcal{R}(C)$ if and only if $P \in\left\{A_{1}, A_{2}, A_{3}\right\}$.

Proof. (1) Since $h^{0}(3 P)=2$ by the Riemann-Roch theorem, $|3 P|$ is free if and only if $h^{0}(2 P)=1$.

(2) If the linear system $|3 P|$ is free, then the first assertion follows from Bertini's theorem. If $|3 P|$ is not free, then $|2 P|=\left|K_{C}\right|$ is free and thus we can take distinct three points $A_{1}:=P, A_{2}, A_{3} \in C$ such that $2 P \sim A_{2}+A_{3}$. Suppose that $3 P \sim$ $\sum_{i=1}^{3} A_{i}$. If $P \in \mathcal{R}(C)$, we have $P \in\left\{A_{1}, A_{2}, A_{3}\right\}$ since $|3 P|$ has a base point $P$. If $P \in\left\{A_{1}, A_{2}, A_{3}\right\}$, then $h^{0}(2 P)=2$.

We always assume that $M_{X}=Z_{X}$ in the rest of this section and use the notation above: notice that $h^{0}\left(D_{2}\right)=1$ and $D_{2} \sim P_{4} \in C \backslash\left\{P_{1}, P_{2}, P_{3}\right\}$, and that $h^{0}(D) \geq$ $\operatorname{deg} D-1$ for any divisor $D$ on $C$ by the Riemann-Roch theorem.

Let $H(\Gamma, t)=\sum_{n \geq 0} c_{n} t^{n}$ denote the Hilbert series associated with a singularity $\left(V^{\prime}, o\right) \in \overline{\mathcal{X}}(\Gamma)$ with $p_{g}\left(V^{\prime}, o\right)=p_{g}(\Gamma)$. As we have seen in (4.7),

$$
\sum_{n \geq 0} c_{n} t^{n}=1+t^{2}+t^{3}+2 t^{4}+t^{5}+2 t^{6}+2 t^{7}+\cdots
$$


We have the following:

$$
\begin{gathered}
h^{0}\left(D_{n}\right)=c_{n} \text { for } n=0,1,2,6 \text { and } n \geq 8, \\
h^{0}\left(D_{3}\right), h^{0}\left(D_{5}\right) \in\{0,1\}, \quad h^{0}\left(D_{4}\right), h^{0}\left(D_{7}\right) \in\{1,2\} .
\end{gathered}
$$

We classify those singularities; they are divided into the following cases:

I. $h^{0}\left(D_{3}\right)=1$.

II. $h^{0}\left(D_{3}\right)=0$ and $h^{0}\left(D_{4}\right)=2$.

III. $h^{0}\left(D_{3}\right)=0$ and $h^{0}\left(D_{4}\right)=1$.

We shall eventually have six cases as seen in Table 2 .

Proposition 4.13. Assume that $M_{X}=Z_{X}$. If $h^{0}\left(D_{3}\right)=1$, then $(V, o)$ is not Gorenstein, $p_{g}(V, o)=8, \operatorname{mult}(V, o)=3, \operatorname{embdim}(V, o)=4$, and

$$
H(V, t)=1+t^{2}+t^{3}+t^{4}+t^{5}+2 t^{6}+t^{7}+\cdots=\frac{1+t^{8}+t^{10}}{\left(1-t^{2}\right)\left(1-t^{3}\right)} .
$$

Furthermore, the $\mathbb{C}$-algebra $R$ is generated by homogeneous elements of degree $2,3,8,10$. Note that $(V, o)$ has the minimal multiplicity among the singularities in $\mathcal{X}(\Gamma)$ (see Remark 4.11).

Proof. We have $h^{0}\left(D_{5}\right)=1$, since $h^{0}\left(D_{2}\right)=h^{0}\left(D_{3}\right)=1$. Since $D_{2} \sim P_{4}$ and $D_{3} \sim 0$, by a similar argument as in the proof of Proposition 4.7 we have that

$$
3 Q \sim 2 \sum_{i=1}^{3} P_{i}, \quad Q \sim 2 P_{4} \sim D_{4} \sim D_{7}, \quad 3 P_{4} \sim \sum_{i=1}^{3} P_{i} .
$$

In particular, $h^{0}\left(D_{4}\right)=h^{0}\left(D_{7}\right)$. By Proposition $4.10(2), \operatorname{mult}(V, o)=3$.

Suppose that $h^{0}\left(D_{4}\right)=2$. Then $(V, o)$ is Gorenstein by Lemma 4.4. Therefore, $\operatorname{embdim}(V, o) \leq \operatorname{mult}(V, o)=3$ by Theorem 4.2. Then $R$ is generated by $x \in R_{2}$, $y \in R_{3}$ and $z \in R_{4}$ as $\mathbb{C}$-algebra $R$ with equation $y^{2}+x z=0$ (cf. the proof of Proposition $4.9(3))$; however, this implies that $(V, o)$ is rational. Hence $h^{0}\left(D_{4}\right)=1$. Then $(V, o)$ is not Gorenstein by Lemma 4.4 , and therefore $(V, o)$ is not hypersurface. Thus, $\operatorname{embdim}(V, o)=4$ by Theorem 4.2. Since $H(\Gamma, t)-H(V, t)=t^{4}+t^{7}$, we have $p_{g}(\Gamma)-p_{g}(V, o)=2$ by Proposition 2.4. Since $x, y$ form a regular sequence of $R$, the Hilbert series of $R /(x, y)$ is $H(V, t)\left(1-t^{2}\right)\left(1-t^{3}\right)=1+t^{8}+t^{10}$. Then we easily see the degrees of generators.

Remark 4.14. By Lemma 4.12, we can take distinct points $P_{1}, \ldots, P_{4} \in C$ such that $3 P_{4} \sim \sum_{i=1}^{3} P_{i}$ and $2 P_{4} \nsim K_{C}$. Let $Q=2 P_{4}$. Then we have

$$
D_{2} \sim P_{4}, \quad D_{3} \sim 2\left(3 P_{4}-\sum_{i=1}^{3} P_{i}\right) \sim 0, \quad h^{0}\left(D_{4}\right)=h^{0}\left(D_{7}\right)=h^{0}\left(2 P_{4}\right)=1,
$$

and $M_{X}=Z_{X}$ by Proposition 4.10. Hence we have a singularity $(V, o) \in \overline{\mathcal{X}}(\Gamma)$ satisfying all the conditions in Proposition 4.13.

Next we consider the case $h^{0}\left(D_{3}\right)=0$. Since $D_{2} \sim P_{4}$, the following three conditions are equivalent (cf. the proof of Proposition 4.7):
(1) $h^{0}\left(D_{3}\right)=0$,
(2) $3 Q \nsim 2 \sum_{i=1}^{3} P_{i}$
(3) $Q \nsim 2 P_{4}$.

Let $x \in R_{2} \backslash\{0\}$. We will compute the embedding dimension of $(V, o)$ via the curve singularity $(V(x), o)$, where $V(x)=\{x=0\} \subset V$. Let $H(V(x), t)=$ $\sum_{n \geq 0} d_{i} t^{i}$ denote the Hilbert series of $R /(x)$. 
Lemma 4.15. The curve $V(x)$ is irreducible and the set $\Gamma_{x}:=\left\{n \in \mathbb{Z}_{\geq 0} \mid d_{n} \neq 0\right\}$ is a numerical semigroup. If $\Gamma_{x}=\left\langle m_{1}, \ldots, m_{e}\right\rangle$, then

$$
\operatorname{embdim}(V, o)-1=\operatorname{embdim}(V(x), o) \leq e .
$$

Proof. Let $H \subset X$ be as in the proof of Proposition 4.10. Then $H$ is irreducible and nonsingular since $E H=1$, and hence the induced map $H \rightarrow V(x)$ is the normalization. If $h \in R \backslash(x)$ is a homogeneous element, then the order of $\left.h\right|_{V(x)}$ at $o \in V(x)$ coincides with the order of vanishing of $h$ along $E_{0}$, that is, $\operatorname{deg} h$. Hence $\Gamma_{x}$ coincides with the so-called semigroup of values of the curve singularity $(V(x), o)$. Then the inequality is well-known.

In the following, it will be useful to notice that the Frobenius number of $\langle a, b\rangle$ is $(a-1)(b-1)-1$.

Proposition 4.16. Assume that $M_{X}=Z_{X}$. If $h^{0}\left(D_{3}\right)=0$ and $h^{0}\left(D_{4}\right)=2$, then $(V, o)$ is not Gorenstein and $\operatorname{mult}(V, o)=4$.

(1) If $h^{0}\left(D_{5}\right)=1$, then $p_{g}(V, o)=8, \operatorname{embdim}(V, o)=4$,

$$
H(V, t)=1+t^{2}+2 t^{4}+t^{5}+2 t^{6}+t^{7}+\cdots=\frac{1+t^{5}+t^{10}+t^{11}}{\left(1-t^{2}\right)\left(1-t^{4}\right)},
$$

and $\mathbb{C}$-algebra $R$ is generated by homogeneous elements of degree $2,4,5,11$.

(2) If $h^{0}\left(D_{5}\right)=0$, then $p_{g}(V, o)=7, \operatorname{embdim}(V, o)=5$,

$$
H(V, t)=1+t^{2}+2 t^{4}+2 t^{6}+t^{7}+\cdots=\frac{1+t^{7}+t^{9}+t^{10}}{\left(1-t^{2}\right)\left(1-t^{4}\right)}
$$

and $\mathbb{C}$-algebra $R$ is generated by homogeneous elements of degree $2,4,7,9,10$.

Proof. We have that $D_{4} \sim 2 P_{4} \sim K_{C}$ and $D_{4} \not D_{7}$. Hence $h^{0}\left(D_{7}\right)=1$ and $(V, o)$ is not Gorenstein by Lemma 4.4. Therefore, $\operatorname{embdim}(V, o) \geq 4$. Since $H^{0}\left(D_{4}\right)$ has no base points, we have $\operatorname{mult}(V, o)=4$ by Proposition 4.10 , and $\operatorname{embdim}(V, o) \leq 5$ by Theorem 4.2. Take homogeneous element $y \in R_{4}$ such that $x$ and $y$ belong to a minimal set of homogeneous generators of $\mathbb{C}$-algebra $R$. Then $x, y$ form a regular sequence of $R$ and the Hilbert series of $R /(x, y)$ is $H^{\prime}(t):=H(V, t)\left(1-t^{2}\right)\left(1-t^{4}\right)$.

(1) Assume that $h^{0}\left(D_{5}\right)=1$. We have $H(V, t)=H(\Gamma, t)-\left(t^{3}+t^{7}\right)$ and $p_{g}(V, o)=$ $p_{g}(\Gamma)-2$ by Proposition 2.4 (2). Since

$$
H(V(x), t)=H(V, t)\left(1-t^{2}\right)=1+t^{4}+t^{5}+t^{8} \sum_{i \geq 0} t^{i}
$$

we have $\Gamma_{x}=\langle 4,5,11\rangle$. It follows from Lemma 4.15 that $\operatorname{embdim}(V, o)=4$. Since $H^{\prime}(t)=1+t^{5}+t^{10}+t^{11}$, we obtain the degrees of homogeneous generators of $R$.

(2) Assume that $h^{0}\left(D_{5}\right)=0$. Then $H(V, t)=H(\Gamma, t)-\left(t^{3}+t^{5}+t^{7}\right), H(V(x), t)=$ $1+t^{4}+t^{7} \sum_{i \geq 0} t^{i}$, and $H^{\prime}(t)=1+t^{7}+t^{9}+t^{10}$. Thus, we obtain the assertion by a similar argument as above.

Remark 4.17. Let $\mathcal{R}(C)$ and $\sigma$ be as in Notation 4.5. Suppose that $P_{4} \in \mathcal{R}(C)$ and $P_{5} \in C \backslash \mathcal{R}(C)$.

(1) Let $Q=P_{4}+P_{5}$. Then $\left|2 Q-P_{4}\right|$ is free since $h^{0}\left(P_{4}+2 P_{5}\right)=2>h^{0}\left(2 P_{5}\right)=$ $h^{0}\left(P_{4}+P_{5}\right)$. Thus, there exist distinct points $P_{1}, P_{2}, P_{3} \in C \backslash\left\{P_{4}\right\}$ such that $2 Q-P_{4} \sim P_{1}+P_{2}+P_{3}$. We set $D=Q-\frac{1}{2} \sum_{i=1}^{3} P_{i}$. Then

$$
\begin{aligned}
D_{2} & \sim P_{4}, \quad D_{3} \sim 2 D_{2}-Q \sim P_{4}-P_{5} \nsim 0, \quad D_{4} \sim K_{C}, \\
D_{5} & \sim 3 D_{2}-Q \sim 2 P_{4}-P_{5} \sim\left(P_{5}+\sigma\left(P_{5}\right)\right)-P_{5}=\sigma\left(P_{5}\right) .
\end{aligned}
$$


Therefore, we have a singularity satisfying the condition of Proposition 4.16 (1).

(2) Let $Q=4 P_{4}-2 P_{5}$. If $\left|2 Q-P_{4}\right|$ has a base point $P_{0}$, then $K_{C} \sim 2 Q-P_{4}-P_{0} \sim$ $7 P_{4}-4 P_{5}-P_{0}$, and thus $5 P_{4} \sim 4 P_{5}+P_{0}$. However, since $\left|5 P_{4}\right|$ has a base point $P_{4}$, we have $4 P_{4} \sim 4 P_{5}$; this is impossible. Hence $\left|2 Q-P_{4}\right|$ is free and there exist distinct points $P_{1}, P_{2}, P_{3} \in C \backslash\left\{P_{4}\right\}$ such that $2 Q-P_{4} \sim P_{1}+P_{2}+P_{3}$. Then

$$
\begin{gathered}
D_{2} \sim P_{4}, \quad D_{3} \sim 2 P_{5}-2 P_{4} \not 0, \quad D_{4} \sim K_{C}, \\
D_{5} \sim 2 P_{5}-P_{4}, \quad h^{0}\left(2 P_{5}-P_{4}\right)=0 .
\end{gathered}
$$

Hence we have a singularity satisfying the condition of Proposition 4.16 (2).

Proposition 4.18. Assume that $M_{X}=Z_{X}$. If $h^{0}\left(D_{3}\right)=0$ and $h^{0}\left(D_{4}\right)=$ $h^{0}\left(D_{5}\right)=1$, then $\operatorname{mult}(V, o)=\operatorname{embdim}(V, o)=5$.

(1) If $h^{0}\left(D_{7}\right)=2$, then $(V, o)$ is Gorenstein, $p_{g}(V, o)=8$,

$H(V, t)=1+t^{2}+t^{4}+t^{5}+2 t^{6}+2 t^{7}+\cdots=\frac{1+t^{6}+t^{7}+t^{8}+t^{14}}{\left(1-t^{2}\right)\left(1-t^{5}\right)}$,

and $\mathbb{C}$-algebra $R$ is generated by homogeneous elements of degree $2,5,6,7,8$.

(2) If $h^{0}\left(D_{7}\right)=1$, then $(V, o)$ is not Gorenstein, $p_{g}(V, o)=7$,

$H(V, t)=1+t^{2}+t^{4}+t^{5}+2 t^{6}+t^{7}+\cdots=\frac{1+t^{6}+t^{8}+t^{9}+t^{12}}{\left(1-t^{2}\right)\left(1-t^{5}\right)}$,

and $\mathbb{C}$-algebra $R$ is generated by homogeneous elements of degree $2,5,6,8,9$.

Proof. The proof is similar to that of Proposition 4.16. We have $R_{4}=R_{2}^{2}$ and $D_{4} \sim 2 P_{4} \nsim K_{C}$. Since $D_{3} \nsim 0$ and $h^{0}\left(D_{5}\right)=1$, there exists a point $P_{5} \in C$ such that $D_{5} \sim P_{5} \neq P_{4}$ (note that $\left.D_{2} \not D_{2}+D_{3}=D_{5}\right)$. Therefore, $\operatorname{mult}(V, o)=5$ by Proposition $4.10(2)$. Let $y \in R_{5} \backslash\{0\}$. Then the Hilbert series of $R /(x, y)$ is $H^{\prime}(t):=H(V, t)\left(1-t^{2}\right)\left(1-t^{5}\right)$. From Lemma $4.4,(V, o)$ is Gorenstein if and only if $h^{0}\left(D_{7}\right)=2$.

(1) Assume that $h^{0}\left(D_{7}\right)=2$. We have $H(V, t)=H(\Gamma, t)-\left(t^{3}+t^{4}\right)$ and $H^{\prime}(t)=1+t^{6}+t^{7}+t^{8}+t^{14}$. Hence $p_{g}(V, o)=p_{g}(\Gamma)-2$ by Proposition 2.4 and $\operatorname{embdim}(V, o)=5$ by Theorem $4.2(2)$. Therefore, $R$ is generated by homogeneous elements of degree $2,5,6,7,8$.

(2) Assume that $h^{0}\left(D_{7}\right)=1$. We have $H(V, t)=H(\Gamma, t)-\left(t^{3}+t^{4}+t^{7}\right)$, $H^{\prime}(t)=1+t^{6}+t^{8}+t^{9}+t^{12}, H(V, t)\left(1-t^{2}\right)=1+t^{5}+t^{6}+t^{8} \sum_{i \geq 0} t^{i}$, and $\Gamma_{x}=\langle 5,6,8,9\rangle$. Hence we obtain the assertion by similar arguments as above.

The following proposition shows the existence and the property of $D$ corresponding to the singularities in Proposition 4.18 (1).

Proposition 4.19. We have the following.

(1) There exist points $P_{1}, \ldots, P_{4} \in C$ and an effective divisor $Q$ of degree two on $C$ which satisfy the condition

(C1) $P_{1}, \ldots, P_{4}$ are distinct, $2 Q \sim \sum_{i=1}^{4} P_{i}, 2 P_{4} \nsim K_{C}, 4 P_{4} \sim Q+K_{C}$.

(2) Let $P_{1}, \ldots, P_{4}$ and $Q$ be as above, and let $D=Q-\frac{1}{2} \sum_{i=1}^{3} P_{i}$. Then the condition (C1) is satisfied if and only if $M_{X}=Z_{X}$ and $h^{0}\left(D_{3}\right)=0$, $h^{0}\left(D_{4}\right)=h^{0}\left(D_{5}\right)=1, h^{0}\left(D_{7}\right)=2$.

Proof. (1) Assume that $\mathcal{R}(C)$ and $\sigma$ be as in Notation 4.5. Let $P_{4} \in C$ satisfies $3\left(P_{4}-\sigma\left(P_{4}\right)\right) \nsim 0$. Then $2 P_{4} \nsim K_{C}$, because $P_{4} \notin \mathcal{R}(C)$. Since $\operatorname{deg}\left(4 P_{4}-K_{C}\right) \geq 2$, there exists an effective divisor $Q$ on $C$ such that $4 P_{4}-K_{C} \sim Q$. Since $\operatorname{deg}(2 Q-$ 
$\left.P_{4}\right)=3$, we have $h^{0}\left(2 Q-P_{4}\right)=2$. If the linear system $\left|2 Q-P_{4}\right|$ is free, then we have distinct three points $P_{1}, P_{2}, P_{3} \in C \backslash\left\{P_{4}\right\}$ such that $2 Q \sim \sum_{i=1}^{4} P_{i}$. If $\left|2 Q-P_{4}\right|$ has a base point $G \in C$, then $2 Q-P_{4}-G \sim K_{C}$. If $G=P_{4}$, we have $2 Q \sim 2 P_{4}+K_{C}$. Since $4 P_{4} \sim Q+K_{C}$, we have $Q+2 P_{4} \sim 2 K_{C} \sim Q+\sigma(Q)$, and hence $2 P_{4} \sim \sigma(Q)$. However, $4 P_{4} \sim Q+K_{C} \sim \sigma\left(2 P_{4}\right)+\sigma\left(P_{4}\right)+P_{4}$; it contradicts that $3\left(P_{4}-\sigma\left(P_{4}\right)\right) \nsim 0$. Therefore, $G \neq P_{4}$. We can take $P_{1} \in C$ so that $P_{1}, P_{2}:=\sigma\left(P_{1}\right), P_{3}:=G, P_{4}$ are distinct. Then $2 Q-P_{4} \sim K_{C}+P_{3} \sim P_{1}+P_{2}+P_{3}$.

(2) Assume that (C1) is satisfied. By Proposition 4.10 (1), we have $M_{X}=Z_{X}$ since $D_{2}=2 Q-\sum_{i=1}^{3} P_{i} \sim P_{4}$. We also have

$$
\begin{gathered}
D_{3} \sim 2 P_{4}-Q \nsim 0, \quad D_{4} \sim 2 P_{4} \nsim K_{C}, \\
D_{5} \sim 3 P_{4}-Q \sim K_{C}-P_{4} \sim P_{4}+\sigma\left(P_{4}\right)-P_{4}=\sigma\left(P_{4}\right), \\
D_{7} \sim 4 P_{4}-Q \sim K_{C} .
\end{gathered}
$$

Thus, we obtain that $\left(h^{0}\left(D_{3}\right), h^{0}\left(D_{4}\right), h^{0}\left(D_{5}\right), h^{0}\left(D_{7}\right)\right)=(0,1,1,2)$.

The converse follows from the arguments above.

Remark 4.20. We take distinct points $P_{4}, P_{5} \in C \backslash \mathcal{R}(C)$ such that $P_{4}+P_{5} \not K_{C}$ and $2\left(2 P_{4}-P_{5}\right) \nsim K_{C}$, and let $Q=3 P_{4}-P_{5}$. Then $P_{4}$ is not a basepoint of $\left|2 Q-P_{4}\right|$. As in the proof of Proposition 4.19, we obtain distinct points $P_{1}, P_{2}, P_{3} \in C \backslash\left\{P_{4}\right\}$ such that $2 Q-P_{4} \sim P_{1}+P_{2}+P_{3}$. Then we have

$$
\begin{gathered}
D_{2} \sim P_{4}, \quad h^{0}\left(D_{3}\right)=h^{0}\left(P_{5}-P_{4}\right)=0, \quad h^{0}\left(D_{4}\right)=h^{0}\left(2 P_{4}\right)=1, \\
h^{0}\left(D_{5}\right)=h^{0}\left(P_{5}\right)=1, \quad h^{0}\left(D_{7}\right)=h^{0}\left(P_{4}+P_{5}\right)=1 .
\end{gathered}
$$

Hence there exists a singularity satisfying the conditions of Proposition 4.18 (2).

Proposition 4.21. Assume that $M_{X}=Z_{X}$. If $h^{0}\left(D_{3}\right)=0, h^{0}\left(D_{4}\right)=1, h^{0}\left(D_{5}\right)=$ 0 . Then $(V, o)$ is not Gorenstein, $h^{0}\left(D_{7}\right)=1, p_{g}(V, o)=6$, $\operatorname{mult}(V, o)=6$, $\operatorname{embdim}(V, o)=7$,

$$
H(V, t)=1+t^{2}+t^{4}+2 t^{6}+t^{7}+\cdots=\frac{1+t^{7}+t^{8}+t^{9}+t^{10}+t^{11}}{\left(1-t^{2}\right)\left(1-t^{6}\right)}
$$

and $\mathbb{C}$-algebra $R$ is generated by homogeneous elements of degree 2, 6, 7, 8, 9, 10, 11 .

Proof. Since $D_{4} \sim 2 P_{4} \nsim K_{C}$ and $D_{6} \sim 3 P_{4}, H^{0}\left(D_{6}\right)$ is free (cf. Lemma 4.12). Hence we have $\operatorname{mult}(V, o)=6$ by Proposition $4.10(2)$ and $\operatorname{embdim}(V, o) \leq 7$ by Theorem 4.2. Take a homogeneous element $y \in R_{6}$ such that $x$ and $y$ belong to a minimal set of homogeneous generators of $\mathbb{C}$-algebra $R$. Then $x, y$ form a regular sequence of $R$ and the Hilbert series of $R /(x, y)$ is $H^{\prime}(t):=H(V, t)\left(1-t^{2}\right)\left(1-t^{6}\right)$.

If $h^{0}\left(D_{7}\right)=2$, then $H^{\prime}(t)=1+2 t^{7}+t^{8}+t^{10}+t^{11}-t^{13}+t^{15}$ has a negative coefficient; it is a contradiction. Hence we have $h^{0}\left(D_{7}\right)=1$. Then $H(V, t)=$ $H(\Gamma, t)-\left(t^{3}+t^{4}+t^{5}+t^{7}\right), H^{\prime}(t)=1+t^{7}+t^{8}+t^{9}+t^{10}+t^{11}$. Hence $p_{g}(V, o)=p_{g}(\Gamma)-4$, $\operatorname{embdim}(V, o)=7$ and $\mathbb{C}$-algebra $R$ is generated by homogeneous elements of degree $2,6,7,8,9,10,11$.

Remark 4.22. Let $P_{4}, P_{5} \in C \backslash \mathcal{R}(C)$ be distinct points such that $P_{4}+P_{5} \nsim K_{C}$. Let $Q=P_{4}+P_{5}$. Then $\left|2 Q-P_{4}\right|$ is free because $h^{0}\left(P_{4}+P_{5}\right)=h^{0}\left(2 P_{5}\right)=1$. Hence there exist distinct three points $P_{1}, P_{2}, P_{3} \in C \backslash\left\{P_{4}\right\}$ such that $2 Q-P_{4} \sim P_{1}+P_{2}+P_{3}$. 
Then we have

$$
\begin{gathered}
h^{0}\left(D_{3}\right)=h^{0}\left(P_{4}-P_{5}\right)=0, \quad h^{0}\left(D_{4}\right)=h^{0}\left(2 P_{4}\right)=1, \\
h^{0}\left(D_{5}\right)=h^{0}\left(2 P_{4}-P_{5}\right)<h^{0}\left(2 P_{4}\right)=1, \\
h^{0}\left(D_{7}\right)=h^{0}\left(3 P_{4}-P_{5}\right)<h^{0}\left(3 P_{4}\right)=2 .
\end{gathered}
$$

Therefore, we have a singularity of Proposition 4.21.

For reader's convenience, we provide a table of the conditions for the PinkhamDemazure divisors $D=Q-\sum_{i=1}^{3} \frac{1}{2} P_{i}$ which induce the singularities discussed in this subsection; for each case, $\mathcal{R}=\mathcal{R}(C)$, four points $P_{1}, \ldots, P_{4} \in C$ are distinct, and $P_{1}+P_{2}+P_{3} \sim 2 Q-P_{4}$.

\begin{tabular}{cccl}
\hline \hline$p_{g}$ & mult & embdim & Pinkham-Demazure divisor \\
\hline 8 & 3 & 4 & $Q=2 P_{4}, P_{4} \notin \mathcal{R}$ \\
8 & 4 & 4 & $Q=P_{4}+P_{5}, P_{4} \in \mathcal{R}, P_{5} \notin \mathcal{R}$ \\
7 & 4 & 5 & $Q=4 P_{4}-2 P_{5}, P_{4} \in \mathcal{R}, P_{5} \notin \mathcal{R}$ \\
8 & 5 & 5 & $Q=4 P_{4}-K_{C}, P_{4} \notin \mathcal{R}$ \\
7 & 5 & 5 & $Q=3 P_{4}-P_{5}, P_{4} \notin \mathcal{R}, P_{5} \notin \mathcal{R}, P_{4} \neq P_{5}$, \\
& & & $P_{4}+P_{5} \nsim K_{C}, 2\left(2 P_{4}-P_{5}\right) \nsim K_{C}$ \\
6 & 6 & 7 & $Q=P_{4}+P_{5}, P_{4} \notin \mathcal{R}, P_{5} \notin \mathcal{R}, P_{4} \neq P_{5}, P_{4}+P_{5} \nsim K_{C}$ \\
\hline \hline
\end{tabular}

TABle 3. Singularities with $M_{X}=Z_{X}$ and Pinkham-Demazure divisors

Remark 4.23. Taking a general Pinkham-Demazure divisor $D=Q-\sum_{i=1}^{3} \frac{1}{2} P_{i}$, we have a singularity $(V, o) \in \overline{\mathcal{X}}(\Gamma)$ with $H(V, t)=1+t^{4}+2 t^{6}+t^{7}+\cdots$ and that $p_{g}(V, o)=5$. Recall that $p_{a}(V, o)=5$ (see Section 4.1). Therefore, we have the equality $p_{a}(V, o)=\min \left\{p_{g}(W, o) \mid(W, o) \in \mathcal{X}(\Gamma)\right\}$, and this is realized by a weighted homogeneous singularity (cf. Theorem 2.6).

\section{REFERENCES}

[1] Shreeram Shankar Abhyankar, Local rings of high embedding dimension, Amer. J. Math. 89 (1967), 1073-1077.

[2] Michael Artin, On isolated rational singularities of surfaces, Amer. J. Math. 88 (1966), 129-136.

[3] Wolf P. Barth, Klaus Hulek, Chris A. M. Peters, and Antonius Van de Ven, Compact complex surfaces, second ed., Ergebnisse der Mathematik und ihrer Grenzgebiete. 3. Folge., vol. 4, Springer-Verlag, Berlin, 2004.

[4] Lev Birbrair, Walter D. Neumann, and Anne Pichon, The thick-thin decomposition and the bilipschitz classification of normal surface singularities, Acta Math. 212 (2014), no. 2, 199256.

[5] David Joseph Dixon, The fundamental divisor of normal double points of surfaces, Pacific J. Math. 80 (1979), no. 1, 105-115.

[6] Shiro Goto and Kei-ichi Watanabe, On graded rings. I, J. Math. Soc. Japan 30 (1978), no. 2, 179-213.

[7] Kazuhiro Konno, Certain normal surface singularities of general type, Methods Appl. Anal. 24 (2017), no. 1, 71-97.

[8] Kazuhiro Konno and Daisuke Nagashima, Maximal ideal cycles over normal surface singularities of Brieskorn type, Osaka J. Math. 49 (2012), no. 1, 225-245. 
[9] Henry B. Laufer, On minimally elliptic singularities, Amer. J. Math. 99 (1977), no. 6, 12571295.

[10] L Lifting cycles to deformations of two-dimensional pseudoconvex manifolds, Trans. Amer. Math. Soc. 266 (1981), no. 1, 183-202.

[11] _ Tangent cones for deformations of two-dimensional quasi-homogeneous singularities, Singularities (Iowa City, IA, 1986), Contemp. Math., vol. 90, Amer. Math. Soc., Providence, RI, 1989, pp. 183-197.

[12] Fan-Ning Meng and Tomohiro Okuma, The maximal ideal cycles over complete intersection surface singularities of Brieskorn type, Kyushu J. Math. 68 (2014), no. 1, 121-137.

[13] David Mumford, The topology of normal singularities of an algebraic surface and a criterion for simplicity, Inst. Hautes Études Sci. Publ. Math. (1961), no. 9, 5-22.

[14] András Némethi, "Weakly" elliptic Gorenstein singularities of surfaces, Invent. Math. 137 (1999), no. 1, 145-167.

[15] András Némethi, Lattice cohomology of normal surface singularities, Publ. Res. Inst. Math. Sci. 44 (2008), no. 2, 507-543.

[16] (2012), no. 4, 2503-2524.

[17] András Némethi and Tomohiro Okuma, The embedding dimension of weighted homogeneous surface singularities, J. Topol. 3 (2010), no. 3, 643-667.

[18] András Némethi and Tomohiro Okuma, Analytic singularities supported by a specific integral homology sphere link, Methods Appl. Anal. 24 (2017), no. 2, 303-320.

[19] András Némethi and Baldur Sigurdsson, The geometric genus of hypersurface singularities, J. Eur. Math. Soc. (JEMS) 18 (2016), no. 4, 825-851.

[20] W. D. Neumann, A calculus for plumbing applied to the topology of complex surface singularities and degenerating complex curves, Trans. Amer. Math. Soc. 268 (1981), no. 2, 299-344.

[21] Tomohiro Okuma, Numerical Gorenstein elliptic singularities, Math. Z. 249 (2005), no. 1, $31-62$.

[22] - The geometric genus of splice-quotient singularities, Trans. Amer. Math. Soc. 360 (2008), no. 12, 6643-6659.

[23] Henry Pinkham, Normal surface singularities with $C^{*}$ action, Math. Ann. 227 (1977), no. 2, 183-193.

[24] Miles Reid, Chapters on algebraic surfaces, Complex algebraic geometry, IAS/Park City Math. Ser., vol. 3, Amer. Math. Soc., Providence, RI, 1997, pp. 3-159.

[25] Judith D. Sally, Tangent cones at Gorenstein singularities, Compositio Math. 40 (1980), no. $2,167-175$.

[26] Jean-Pierre Serre, Sur les modules projectifs, Séminaire Dubreil. Algèbre et théorie des nombres 14 (1960-1961), no. 1.

[27] Masataka Tomari, A $p_{g}$-formula and elliptic singularities, Publ. Res. Inst. Math. Sci. 21 (1985), no. 2, 297-354.

[28] Maximal-ideal-adic filtration on $\mathbf{R}^{1} \psi_{*} \mathcal{O}_{\tilde{V}}$ for normal two-dimensional singularities, Complex analytic singularities, Adv. Stud. Pure Math., vol. 8, North-Holland, Amsterdam, 1987, pp. 633-647.

[29] Masataka Tomari and Tadashi Tomaru, The maximal ideal cycles over normal surface singularities with $\mathbb{C}^{*}$-action, Tohoku Math. J. (2) 69 (2017), no. 3, 415-430.

[30] Masataka Tomari and Kei-ichi Watanabe, Filtered rings, filtered blowing-ups and normal two-dimensional singularities with "star-shaped" resolution, Publ. Res. Inst. Math. Sci. 25 (1989), no. 5, 681-740.

[31] Tadashi Tomaru, On Kodaira singularities defined by $z^{n}=f(x, y)$, Math. Z. 236 (2001), no. $1,133-149$.

[32] Philip Wagreich, Elliptic singularities of surfaces, Amer. J. Math. 92 (1970), 419-454.

[33] Kei-ichi Watanabe, Some remarks concerning Demazure's construction of normal graded rings, Nagoya Math. J. 83 (1981), 203-211.

[34] Stephen Shing Toung Yau, On maximally elliptic singularities, Trans. Amer. Math. Soc. 257 (1980), no. 2, 269-329.

Department of Mathematical Sciences, Yamagata University, Yamagata 990-8560, JAPAN.

Email address: okuma@sci.kj.yamagata-u.ac.jp 